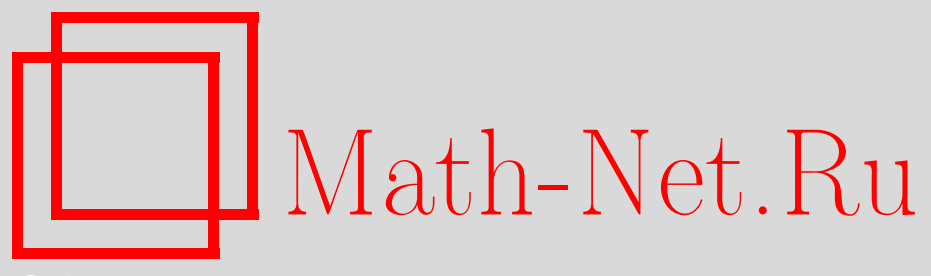

В. Е. Назайкинский, А. Ю. Савин, Б. Ю. Стернин, О гомотопической классификации эллиптических операторов на стратифицированных многообразиях, Изв. РАН. Сер. матем., 2007, том 71, выпуск 6, 91-118

DOI: https://doi.org/10.4213/im781

Использование Общероссийского математического портала Math-Net.Ru подразумевает, что вы прочитали и согласны с пользовательским соглашением http://www . mathnet.ru/rus/agreement

Параметры загрузки:

IP : 107.22 .136 .117

26 апреля 2023 г., 18:10:46

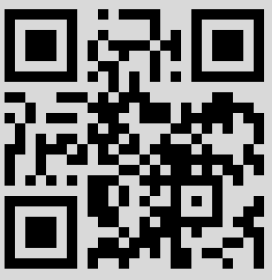


УДК $515.168 .5+517.986 .32$

\author{
В. Е. Назайкинский, А. Ю. Савин, Б. Ю. Стернин
}

\title{
О гомотопической классификации эллиптических операторов на стратифицированных многообразиях
}

\begin{abstract}
Дана гомотопическая классификация эллиптических операторов на стратифицированных многообразиях. Именно, получен изоморфизм множества эллиптических операторов по модулю стабильных гомотопий и группы $K$-гомологий многообразия. В качестве приложения классификации дана явная формула для препятствия типа Атьи-Ботта к постановке фредгольмовых задач в ситуации стратифицированных многообразий.

Билиография: 35 наименований.
\end{abstract}

\section{$\S$ 1. Введение}

В классической работе М. Атьи [1] было замечено, что абстрактные эллиптические операторы на компактном пространстве $X$ (напомним, что так называют фредгольмовы операторы в $C(X)$-модулях, перестановочные с операторами умножения на функции с точностью до компактных операторов) определяют элементы группы $K$-гомологий пространства $X$. Более того, Г. Г. Каспаров [2] и Л. Браун, Р. Дуглас, П. Филмур [3] показали, что можно получить не только элементы $K$-гомологических групп, но и реализацию $K$-гомологий как обобщенной теории гомологий, если профакторизовать множество абстрактных эллиптических операторов по отношению эквивалентности, задаваемому стабильной гомотопией.

Оказывается, однако, что для гладких многообразий группу $K$-гомологий можно получить, если вместо абстрактных операторов ограничиться дифбереницальными (псевдодифференииальными) операторами, естественными в теории уравнений с частными производными. Более того, если при этом многообразие дополнительно имеет spin $^{\mathrm{c}}$-структуру, то достаточно рассматривать только (скрученные) операторы Дирака. Этот пример служит мотивировкой постановки естественной задачи сравнения группы $K$-гомологий и группы, порожденной эллиптическими псевдодифференциальными операторами (ПДО) для негладких пространств (ср. с проблемой Зингера в [4]), в частности для стратифицированных многообразий.

$\mathrm{K}$ настоящему времени решение этой задачи известно в некоторых частных случаях. Так, для многообразий с изолированными особенностями классификация общих эллиптических ПДО в терминах $K$-гомологий была установлена

Работа выполнена при частичной поддержке РФФИ (гранты № 05-01-00928, 06-01-00098), Программы Президента РФ "Молодые кандидаты” (грант МK-1713.2005.1), а также гранта DFG 436 RUS 113/849/0-1® “K-теория и некоммутативная геометрия стратифицированных многообразий". 
в [5]. Этот результат был независимо доказан с использованием группоидов и $K K$-теории в [6], [7]. Для стратифицированных многообразий с двумя стратами классификация реберно-вырождающихся эллиптических операторов получена в [8], [9].

Исчисление ПДО на общих стратифицированных многообразиях не является чем-то новым. Оно было построено, например, в [10], а кроме того, в [11], [12] в рамках предложенного в [13] общего подхода к построению ПДО, ассоциированных с заданной алгеброй Ли векторных полей, при использовании аппарата теории группоидов. Однако результаты о гомотопической классификации эллиптических операторов на таких многообразиях до сих пор известны не были. Такие результаты устанавливаются в настоящей работе.

Основная теорема, доказанная в настоящей работе, утверждает, что для компактного стратифицированного многообразия $X$ с произвольным конечным числом стратов имеет место изоморфизм групп

$$
\operatorname{Ell}(X) \simeq K_{0}(X)
$$

где $\operatorname{Ell}(X)$ - группа, порожденная эллиптическими псевдодифференциальными операторами на многообразии $X$ по модулю стабильных гомотопий, а $K_{0}(X)$ группа четных $K$-гомологий многообразия $X$. Частные случаи этого изоморфизма были получены в цитированных выше работах [5]-[9].

Изоморфизм (1.1) позволяет перенести топологические методы из теории $K$-гомологий в эллиптическую теорию. В качестве примеров приложения топологических методов мы вычисляем препятствие типа Атьи-Ботта к постановке фредгольмовых задач на стратифицированных многообразиях, а также даем обобщение теоремы об инвариантности индекса относительно кобордизма (cм. $\S 8)$.

Кроме указанных приложений к эллиптическим операторам, изоморфизм (1.1) имеет интересную интерпретацию в рамках некоммутативной геометрии. Именно, с точки зрения некоммутативной геометрии алгебра ПДО на стратифицированном многообразии отвечает некоторому группоиду (см. [14], [15]). При этом группа $\operatorname{Ell}(X)$ связана с $K$-группой $C^{*}$-алгебры группоида [12]. Известная гипотеза Баума-Конна [16] утверждает, что последняя изоморфна топологической $K$-группе для классифицирующего пространства группоида (см. [17]). Явные вычисления для простейших стратифицированных многообразий показывают, что $K$-группа классифицирующего пространства изоморфна $K_{0}(X)$ - правой части соотношения (1.1). Интересным представляется дальнейшее сравнение изоморфизма (1.1) с отображением Баума-Конна.

В заключение введения мы хотели бы выразить признательность профессору Т. Факу (Лион), который указал на возможную связь изоморфизма (1.1) с изоморфизмом Баума-Конна для группоидов.

Изложенные здесь результаты были доложены на Международной конференции "Семинар по теории индекса", Мюнстер (4-8 ноября 2005 г.). 


\section{§ 2. Стратифицированные многообразия и ПДО}

В настоящем параграфе мы опишем класс многообразий, на которых будет изучаться эллиптическая теория, и дадим описание класса рассматриваемых псевдодифференциальных операторов. Эти темы хорошо известны (см., например, [10], [18], [19]), поэтому наше изложение будет достаточно кратким.

2.1. Стратифицированные многообразия. Далее будем использовать следующую терминологию. Многообразием с особенностями назовем тройку

$$
\pi: M \rightarrow \mathfrak{M}
$$

где $\mathfrak{M}$ - хаусдорфово пространство, $M$ - многообразие с углами ${ }^{1}$, а $\pi$ - непрерывная проекция. Многообразие $M$ будет называться раздутием многообразия $\mathfrak{M}$. Мы не обсуждаем вопрос о единственности раздутия, а, говоря о многообразии с особенностями, всегда считаем, что зафиксирована тройка $\pi: M \rightarrow \mathfrak{M}$.

Диффеоморфизмом таких многообразий будем называть пару $\left(f: \mathfrak{M}_{1} \rightarrow \mathfrak{M}_{2}\right.$, $\left.\tilde{f}: M_{1} \rightarrow M_{2}\right)$, состоящую из гомеоморфизма $f$ и диффеоморфизма $\tilde{f}$, такую, что коммутативна диаграмма

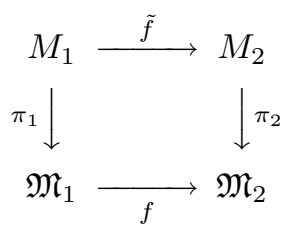

где $\pi_{1}, \pi_{2}$ - естественные проекции. Частным случаем многообразий с особенностями является следующий. Если на многообразии $M$ с углами задана гладкая риманова метрика, невырожденная во внутренности многообразия и, возможно, вырождающаяся на границе, то М определяется как факторпространство многообразия $M$ по отношению эквивалентности, задаваемому метрикой: две точки эквивалентны, если расстояние между ними в метрике равно нулю. (Разумеется, необходимо предполагать хаусдорфовость многообразия $\mathfrak{M}$.)

Опишем интересующий нас класс многообразий с особенностями - стратифицированных многообразий. Это описание будет индуктивным.

ОПРЕДЕЛЕНИЕ 2.1. Филътрачией длинъ $k$ на топологическом пространстве $\mathfrak{M}$ называется последовательность

$$
\mathfrak{M}=\mathfrak{M}_{k} \supset \mathfrak{M}_{k-1} \supset \cdots \supset \mathfrak{M}_{0}
$$

вложенных замкнутых в $\mathfrak{M}$ топологических пространств такая, что каждое $\mathfrak{M}_{j}$ лежит в замыкании множества $\mathfrak{M}_{j+1}^{\circ}=\mathfrak{M}_{j+1} \backslash \mathfrak{M}_{j}, j=0,1, \ldots, k-1$.

ОПРЕДЕЛЕНИЕ 2.2 (базис индукции). Стратифицированным многообразием нулевой длины называется произвольное гладкое многообразие. При этом $M=\mathfrak{M}, \pi=\mathrm{id}$ и раздутие $M$ - гладкое многообразие без края.

\footnotetext{
${ }^{1}$ Напомним, что многообразием размерности $n$ с углами называется хаусдорфово пространство, локально гомеоморфное произведению $\overline{\mathbb{R}}_{+}^{k} \times \mathbb{R}^{n-k}, 0 \leqslant k \leqslant n$, с гладкими функциями перехода между областями такого вида.
} 
ОПРЕДЕЛЕНИЕ 2.3 (шаг индукции). Стратифицированным многообразием длины $k>0$ называется хаусдорфово пространство $\mathfrak{M}$, снабженное фильтрацией $(2.1)$, такое, что:

1) $\mathfrak{M}_{0}$ наделено структурой гладкого многообразия;

2) $\mathfrak{M} \backslash \mathfrak{M}_{0}$ наделено структурой стратифицированного многообразия длины $k-1$ относительно фильтрации

$$
\mathfrak{M} \backslash \mathfrak{M}_{0}=\mathfrak{M}_{k} \backslash \mathfrak{M}_{0} \supset \mathfrak{M}_{k-1} \backslash \mathfrak{M}_{0} \supset \cdots \supset \mathfrak{M}_{1} \backslash \mathfrak{M}_{0}
$$

$3)$ над $\mathfrak{M}_{0}$ задано расслоение со слоем $K_{\Omega}$, где $K_{\Omega}-$ конус с базой $\Omega$, которая является компактным стратифицированным многообразием длины не больше $k-1$, а также задан гомеоморфизм окрестности $U \subset \mathfrak{M}$ множества $\mathfrak{M}_{0}$ на окрестность подрасслоения, образованного вершинами конусов, который на $\mathfrak{M}_{0}$ является тождественным отображением;

4) приведенная в п. 3) структура согласована со структурой из п. 2) на $\mathfrak{M} \backslash \mathfrak{M}_{0}$ в указанном ниже смысле.

Отсюда по индукции вытекает, что:

i) множества $\mathfrak{M}_{j} \backslash \mathfrak{M}_{j-1} \simeq M_{j}^{\circ}$ (открытые страты $)$ - гладкие многообразия ${ }^{2}$ для всех $j=0,1, \ldots, k$;

ii) любой открытый страт $M_{j}^{\circ}, j<k$, имеет окрестность $U_{j} \subset \mathfrak{M}$, гомеоморфную расслоению со слоем $K_{\Lambda_{j}}$, где база конуса $\Lambda_{j}$ является стратифицированным многообразием длины не более $k-j-1$.

Укажем точные формулировки условий 3) и 4).

В условии 3) конус определяется формулой

$$
K_{\Omega}=\left\{\overline{\mathbb{R}}_{+} \times \Omega\right\} /\{\{0\} \times \Omega\} .
$$

Требуется, чтобы функции склейки расслоения со слоем $K_{\Omega}$ оставляли переменную $r \in \overline{\mathbb{R}}_{+}$инвариантной и были индуцированы дифбеоморфизмами стратифицированного многообразия $\Omega$ длины не более $k-1$, т. е. диффеоморфизмами многообразий с особенностями, сохраняющими стратификацию и расслоения окрестностей стратов на конусы. Таким образом, мы фактически требуем, чтобы наше расслоение получалось из некоторого расслоения над $\mathfrak{M}_{0}$ со слоем $\Omega$ путем взятия конуса в каждом слое.

Мы утверждаем, что дополнение $U \backslash \mathfrak{M}_{0}$ имеет естественную структуру стратифицированного многообразия длины не более $k-1$ как открытое подмножество расслоения над $\mathfrak{M}_{0}$ со слоем

$$
K_{\Omega}^{\circ} \simeq \mathbb{R}_{+} \times \Omega
$$

- конусом без вершины. Действительно, локальные тривиализации этого расслоения имеют вид $V \times \mathbb{R}_{+} \times \Omega$, где $V \subset \mathbb{R}^{l}, l=\operatorname{dim} \mathfrak{M}_{0},-$ координатная окрестность на $\mathfrak{M}_{0}$. Таким образом, они являются стратифицированными многообразиями той же длины, что и $\Omega$, т. е. не более $k-1$. В качестве стратов в $V \times \mathbb{R}_{+} \times \Omega$ можно взять $V \times \mathbb{R}_{+} \times \Omega_{j}$, где $\Omega_{j}$ - соответствующие страты в $\Omega$,

2 Для удобства мы полагаем $\mathfrak{M}_{-1}=\varnothing$. Эти множества суть внутренности соответствующих раздутий - гладких многообразий с углами. 
а расслоения окрестностей этих стратов на конусы получаются из расслоений окрестностей соответствующих стратов в $\Omega$ умножением на $V \times \mathbb{R}_{+}$.

Поясним теперь условие 4). В силу предыдущего дополнение $U \backslash \mathfrak{M}_{0}$ оказывается наделенным двумя структурами стратифицированного многообразия, одна из которых получается ограничением соответствующей структуры из $\mathfrak{M} \backslash \mathfrak{M}_{0}$, а другая - из расслоения, и условие согласования заключается в том, чтобы эти две структуры совпадали (т. е. тождественное отображение было диффеоморфизмом).

Наконец, определим раздутие многообразия $\mathfrak{M}$. Пусть $\tilde{\pi}: \widetilde{M} \rightarrow \mathfrak{M} \backslash \mathfrak{M}_{0}-$ раздутие многообразия $\mathfrak{M} \backslash \mathfrak{M}_{0}$ (оно известно по предположению индукции). Раздутие $M$ многообразия $\mathfrak{M}$ получается из $\widetilde{M}$ добавлением некоторого множества "над $\mathfrak{M}_{0}$ ". Поэтому, чтобы описать $M$ и проекцию $\pi: M \rightarrow \mathfrak{M}$, достаточно изучить, что происходит над $U$. Можно считать, что $U$ расслоено над $\mathfrak{M}_{0}$; тогда $\tilde{\pi}^{-1}\left(U \backslash \mathfrak{M}_{0}\right)$ расслоено над $\mathfrak{M}_{0}$ с помощью сквозного отображения

$$
\tilde{\pi}^{-1}\left(U \backslash \mathfrak{M}_{0}\right) \stackrel{\tilde{\pi}}{\rightarrow} U \backslash \mathfrak{M}_{0} \rightarrow \mathfrak{M}_{0} .
$$

Структуру этого расслоения несложно описать в локальных тривиализациях; она имеет вид

$$
V \times \mathbb{R}_{+} \times \widetilde{\Omega} \stackrel{\text { id } \times \text { id } \times p}{\longrightarrow} V \times \mathbb{R}_{+} \times \Omega \rightarrow V,
$$

где $p: \widetilde{\Omega} \rightarrow \Omega$ - раздутие базы конуса. Теперь в этой же локальной тривиализации раздутие $M$ многообразия $\mathfrak{M}$ определим как добавление точки $r=0$ ко второму сомножителю, т. е. как переход от $\mathbb{R}_{+}$к $\overline{\mathbb{R}}_{+} ;$проекция переводит любую точку вида $(v, 0, \omega)$ в $v$.

Теперь смысл определения 2.3 полностью прояснен.

Каждый из стратов $\mathfrak{M}_{j}$ является стратифицированным многообразием (длины $j$ ). Он имеет раздутие $M_{j}$, которое в дальнейшем будем называть замкнуmым стратом, и определена соответствующая проекция $p_{j}: M_{j} \rightarrow \mathfrak{M}_{j}$.

ЗАМЕчАниЕ 2.1. Согласно условию 3) расслоения конусов определены над открытыми стратами $M_{j}^{\circ}$ многообразия $\mathfrak{M}$. Однако, как нетрудно видеть, из условия 4) вытекает, что эти расслоения каноническим образом продолжаются на замкнутые страты $M_{j}$.

Это замечание будет полезно нам в дальнейшем, поскольку операторнозначные символы наших псевдодифференциальных операторов будут определены именно над замкнутыми стратами.

Метрики, меры и пространства $L^{2}$. На стратифицированных многообразиях введем некоторые естественные метрики и меры, относительно которых будут рассматриваться пространства суммируемых с квадратом функций.

Прежде всего дадим индуктивное описание метрик. Это можно сделать следующим образом. На стратифицированном многообразии нулевой длины будем брать произвольную риманову метрику. Далее опишем шаг индукции. Локально на $V \times \mathbb{R}_{+} \times \widetilde{\Omega}$ зададим метрику формулой

$$
d s^{2}=d v^{2}+d r^{2}+r^{2} d \widetilde{\omega}^{2}
$$

где $d \widetilde{\omega}^{2}$ - заданная по предположению индукции метрика на $\widetilde{\Omega}$. Глобально метрика на $\mathfrak{M}$ получается склеиванием с помощью разбиения единицы указанных 
локальных выражений, заданных в окрестности подмногообразия $\mathfrak{M}_{0}$, и метрики $\tilde{d} \rho^{2}$, заданной на стратифицированном многообразии $\mathfrak{M} \backslash \mathfrak{M}_{0}$ по предположению индукции, вне меньшей окрестности этого подмногообразия. Такого рода метрики будем называть метриками с реберным вырождением.

Всякой метрике естественным образом соответствует мера - элемент объема, равный единице на наборе ортогональных векторов единичной длины. В терминах индуктивной формулы (2.2) соответствующая формула для меры имеет вид

$$
d \mathrm{vol}=r^{n} d v d r d \operatorname{vol}_{\Omega},
$$

где $d \mathrm{vol}_{\Omega}$ - известный по предположению индукции элемент объема на $\Omega$, а $n=\operatorname{dim} \Omega-$ размерность многообразия $\Omega$.

Далее все операторы на $\mathfrak{M}$ будем рассматривать в пространстве

$$
L^{2}(\mathfrak{M}) \equiv L^{2}(\mathfrak{M}, d \mathrm{vol})
$$

а на конусе $K_{\Omega}-$ в пространстве

$$
L^{2}\left(K_{\Omega}\right) \equiv L^{2}\left(K_{\Omega}, r^{n} d r d \operatorname{vol}_{\Omega}\right) .
$$

Кокасательное расслоение. На многообразии $\mathfrak{M}$ определим некоторое про-

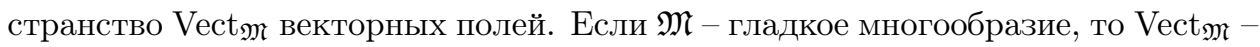
пространство всех векторных полей на $\mathfrak{M}$. Далее определим Vect $_{\mathfrak{M}}$ по индукции локально, считая, что это $C^{\infty}(M)$-модуль. На произведении $V \times \mathbb{R}_{+} \times \widetilde{\Omega}$ пространство Vect $_{\mathfrak{M}}$ состоит из векторных полей вида

$$
\theta=a \frac{\partial}{\partial v}+b \frac{\partial}{\partial r}+\frac{1}{r} \theta_{1}
$$

где $a, b$ - гладкие функции, а $\theta_{1} \in \operatorname{Vect}_{\Omega}$.

Метрика $d s^{2}$ задает $C^{\infty}(M)$-значное спаривание на пространстве Vect $_{\mathfrak{M}}$, а формула

$$
\langle\varphi(\theta), \mu\rangle=d s^{2}(\theta, \mu)
$$

- биекцию $\varphi$ пространства $\operatorname{Vect}_{\mathfrak{M}}$ на некоторый $C^{\infty}(M)$-модуль $\Lambda^{1}(\mathfrak{M}) \subset \Lambda^{1}(M)$ дифференциальных форм на раздутии $M$ многообразия $\mathfrak{M}$ (чтобы убедиться в этом, достаточно усмотреть, что $\operatorname{Vect}_{M} \subset$ Vect $_{\mathfrak{M}}$ и вложение эпиморфно на плотном основном страте).

ОПРЕДЕЛЕНИЕ 2.4. Кокасателъным расслоением $T^{*} \mathfrak{M}$ многообразия $\mathfrak{M}$ называется (существующее по теореме Свана) расслоение над раздутием $M$, сечения которого суть элементы $\Lambda^{1}(\mathfrak{M})$.

ЗАмЕчАниЕ 2.2. Элементы $\Lambda^{1}(\mathfrak{M})$ суть именно те формы, которые обращаются в нуль на слоях проекции $\pi: M \rightarrow \mathfrak{M}$.

ЗАмЕчАниЕ 2.3. Поскольку дифференциальные операторы на стратифицированном многообразии $\mathfrak{M}$ суть полиномы с гладкими (из $\left.C^{\infty}(M)\right)$ коэффициентами от векторных полей из Vect $_{\mathfrak{M}}$, то нетрудно убедиться в том, что их внутренние символы являются гладкими функциями на $T^{*} \mathfrak{M}$.

Аналогичным образом определяются кокасательные расслоения стратов $\mathfrak{M}_{j}$. 
Пространство $C^{\infty}(\mathfrak{M})$. Элементы пространства $C^{\infty}(\mathfrak{M})$ определим как гладкие функции на раздутии $M$ многообразия $\mathfrak{M}$, которые в координатах $(v, r, \omega)$ в окрестности любого страта немаксимальной размерности зависят только от $v$ при достаточно малом $r$.

2.2. Псевдодифференциальные операторы и символы. Опишем алгебру ПДО нулевого порядка на стратифицированном многообразии М. Как уже отмечалось во введении, существует несколько различных изложений теории ПДО на стратифицированных многообразиях. Мы будем пользоваться конструкцией, данной в [21], и для удобства читателя приводим соответствующие определения и факты. Доказательства содержащихся в этом пункте утверждений (кроме предложения 2.2) не приводятся ввиду их громоздкости (их можно найти в [21]).

Будем определять ПДО с параметром $v$ из конечномерного векторного пространства $V$; последовательными (и тривиальными) обобщениями на каждом шаге индукции сначала получается случай, когда ПДО гладко зависят от некоторого дополнительного параметра $x$, а затем и случай, когда ПДО параметризованы точками некоторого конечномерного векторного расслоения над гладким многообразием.

Пренебрежимые семейства. Введем некоторое пространство операторных семейств, по модулю которых будут определяться ПДО. Пусть М - стратифицированное многообразие (возможно, некомпактное). Через $J_{\infty}(V, \mathfrak{M}) \equiv$ $J_{\infty}(\mathfrak{M}) \equiv J_{\infty}$ обозначим пространство гладко зависящих от параметра $v \in V$ операторных семейств

$$
D(v): L^{2}(\mathfrak{M}) \rightarrow L^{2}(\mathfrak{M})
$$

таких, что все операторы $D(v)$ компактны в $L^{2}(\mathfrak{M})$, выполнены оценки

$$
\left\|\frac{\partial^{\beta} D(v)}{\partial v^{\beta}}\right\| \leqslant C_{\beta N}(1+|v|)^{-N}, \quad|\beta|, N=0,1,2, \ldots,
$$

и эти условия остаются справедливыми, если $D(v)$ заменить на произведение

$$
V_{1} \cdots V_{p} D(v) V_{p+1} \cdots V_{p+q}
$$

произвольной длины $p+q \geqslant 0$, где $V_{1}, \ldots, V_{p+q}$ - гладкие векторные поля на $M$, которые вблизи каждого страта в локальных координатах $(x, r, \omega) \in \mathbb{R}^{m} \times \overline{\mathbb{R}}_{+} \times$ $\Omega$ имеют вид $V=(V(x), 0, \widetilde{V}(x, \omega))$, где $V(x)$ - гладкое векторное поле на страте, а $\widetilde{V}(x, \omega)$ - гладко зависящее от $x \in X$ поле с такими же свойствами на многообразии $\Omega$ меньшей длины.

ПДО с параметром на гладких многообразиях. Теперь можно дать определение ПДО. Определение наше является индуктивным, и мы начнем с того, что опишем класс ПДО с параметром на гладких многообразиях.

ОПРЕДЕЛЕНИЕ 2.5. Псевдодифберенииальным оператором с параметром $v \in V$ на гладком многообразии $\mathfrak{M}$ будем называть операторное семейство

$$
D(v): L^{2}(\mathfrak{M}) \rightarrow L^{2}(\mathfrak{M}),
$$


которое представляет собой ПДО нулевого порядка на $\mathfrak{M}$ с параметром $v \in V$ в смысле Аграновича-Вишика. Под символом оператора $D$ (соответствующим единственному страту многообразия $\mathfrak{M})$ будем понимать символ $\sigma(D)(x, \xi, v)$ в смысле Аграновича-Вишика, определенный на пространстве векторного расслоения $T^{*} \mathfrak{M} \times V$ над $\mathfrak{M}$ вне нулевого сечения.

Пространство псевдодифференциальных операторов с параметром $v \in V$ на $\mathfrak{M}$ обозначим через $\Psi(V, \mathfrak{M})$. (Аргумент $V$ не будем выписывать, если $V$ очевидно из контекста или тривиально.)

ПДО с параметрами на стратифицированных многообразиях. Здесь мы определим ПДО индукцией по длине $k$ стратифицированного многообразия. Заметим, что одновременно будут определяться и ПДО, и их символы. Базис индукции - определение 2.5 , пригодное при $k=0,-$ мы уже имеем.

ОПРЕДЕЛЕНИЕ 2.6. Пусть $\mathfrak{M}$ - стратифицированное многообразие длины $k>0$. Гладкое семейство линейных операторов

$$
D(v): L^{2}(\mathfrak{M}) \rightarrow L^{2}(\mathfrak{M})
$$

называется псевдодифференциалъным оператором на $\mathfrak{M}$ ( с параметром $v \in V$ в смысле Аграновича-Вишика), если выполнены следующие условия:

1) если $\varphi, \psi \in C^{\infty}(\mathfrak{M})$ и $\operatorname{supp} \varphi \cap \operatorname{supp} \psi=\varnothing$, то $\psi A \varphi \in J_{\infty}$;

2) оператор $D$ является ПДО с параметром на многообразии ${ }^{3} \mathfrak{M} \backslash \mathfrak{M}_{0}$, а в окрестности $U$ многообразия $\mathfrak{M}_{0}$ оператор $D$ с точностью до элементов из $J_{\infty}(V, \mathfrak{M})$ представи́м в виде

$$
D=P\left(x, r, r v,-i r \frac{\partial}{\partial x}, i r \frac{\partial}{\partial r}+i \frac{n+1}{2}\right), \quad n=\operatorname{dim} \Omega,
$$

где $P(x, r, v, \eta, p) \in \Psi\left(V \times T_{x}^{*} \mathfrak{M}_{0} \times \mathbb{R}, \Omega\right)$ - ПДО с параметрами на $\Omega$, дополнительно гладко зависящий от параметров $x \in \mathfrak{M}_{0}$ и $r \in \overline{\mathbb{R}}_{+}$, причем $P=0$ при $r>r_{0}$, где $r_{0}$ достаточно мало.

Cимволами $\sigma_{j}(D)$ оператора $D$, отвечающими стратам $\mathfrak{M}_{j} \backslash \mathfrak{M}_{j-1}, j>0$, многообразия $\mathfrak{M}$ называются его символы как оператора из $\Psi\left(V, \mathfrak{M} \backslash \mathfrak{M}_{0}\right)$. Символ $\sigma_{k}(D)$ называется внутренним символом оператора D. Cuмволом оператора $D$, отвечающим страту $\mathfrak{M}_{0}$, называется параметризованное точками расслоения $V \times T^{*} \mathfrak{M}_{0}$ над $\mathfrak{M}_{0}$ без нулевого сечения операторное семейство

$$
\sigma_{0}(D)=P\left(x, 0, r v, r \xi, i r \frac{\partial}{\partial r}+i \frac{n+1}{2}\right): L^{2}\left(K_{\Omega}\right) \rightarrow L^{2}\left(K_{\Omega}\right) .
$$

Cимволами $\sigma_{j}\left(\sigma_{0}(D)\right)$ символа $\sigma_{0}(D)$ назовем символы $\sigma_{j}(P(x, 0, v, \eta, p))$ ПДО $P(x, 0, v, \eta, p)$ с параметрами на $\Omega, j=1, \ldots, k$.

Дадим некоторые пояснения формул (2.5) и (2.6). Индукцией по длине многообразия показывается, что псевдодифференциальный оператор $A \in \Psi(V, \mathfrak{M})$ удовлетворяет оценкам

$$
\left\|\frac{\partial^{\alpha} A(v)}{\partial v^{\alpha}}\right\| \leqslant C_{\alpha}(1+|v|)^{-|\alpha|}, \quad|\alpha|=0,1,2, \ldots,
$$

\footnotetext{
${ }^{3}$ Страт $\mathfrak{M}_{0}$ имеет нулевую меру в $\mathfrak{M}$, так что оператор $D$ автоматически интерпретируется как оператор в $L^{2}\left(\mathfrak{M} \backslash \mathfrak{M}_{0}\right)$.
} 
причем все производные, начиная с первой, компактнозначны. Применяя это утверждение для $\mathfrak{M}=\Omega$, получим, что операторное семейство

$$
F(x, t, v, \xi, p)=P\left(x, e^{-t}, v e^{-t}, \xi e^{-t}, p\right)
$$

удовлетворяет оценкам

$$
\begin{gathered}
\left\|\frac{\partial^{\alpha+l+\beta+\gamma+k} F(x, t, v, \xi, p)}{\partial x^{\alpha} \partial t^{l} \partial v^{\beta} \partial \xi^{\gamma} \partial p^{k}}\right\| \leqslant C_{\alpha l \beta \gamma k}(1+|v|+|\xi|)^{-|\beta|-|\gamma|}(1+|p|)^{-k}, \\
|\alpha|+l+|\beta|+|\gamma|+k=0,1,2, \ldots,
\end{gathered}
$$

а операторное семейство $\widetilde{F}(x, t, v, \xi, p)=P\left(x, 0, v e^{-t}, \xi e^{-t}, p\right)$ - оценкам

$$
\begin{aligned}
& \left\|\frac{\partial^{\alpha+l+\beta+\gamma+k} \widetilde{F}(x, t, v, \xi, p)}{\partial x^{\alpha} \partial t^{l} \partial v^{\beta} \partial \xi^{\gamma} \partial p^{k}}\right\| \leqslant C_{\alpha l \beta \gamma k}\left(e^{t}+|v|+|\xi|\right)^{-|\beta|-|\gamma|}(1+|p|)^{-k} \\
& \quad \leqslant C_{\alpha l \beta \gamma k}(|v|+|\xi|)^{-|\beta|-|\gamma|}(1+|p|)^{-k}, \quad|\alpha|+l+|\beta|+|\gamma|+k=0,1,2, \ldots,
\end{aligned}
$$

причем оба семейства имеют компактную вариацию по параметрам $(v, \xi, p)$. Теперь нетрудно показать, что операторы в правых частях (2.5) и (2.6) корректно определены как ПДО в смысле Люк [20]. В самом деле, сделаем замену переменной $r=e^{-t}$, переводящую конус $K_{\Omega}$ в цилиндр $\Omega \times \mathbb{R}$, а оператор $i r \partial / \partial r-$ в оператор $-i \partial / \partial t$. Остается заметить, что оператор $-i \partial / \partial t+i(n+1) / 2$ самосопряжен в пространстве $L^{2}$ на этом цилиндре с весом $e^{-(n+1) t}$, в которое переходит пространство $L^{2}\left(K_{\Omega}\right)$ при такой замене, так что подстановка указанного оператора в качестве операторного аргумента законна. Более того, вновь полученный ПДО удовлетворяет оценкам (2.7), так что шаг индукции завершен.

ЗАмЕчАниЕ 2.4. В силу некомпактности конуса (по переменной $r$ ) операторнозначный символ (2.6) имеет лишь почти компактную вариацию по переменным $(\xi, v)$ (т. е. вариация становится компактной при умножении ее на финитную срезающую функцию по переменной $r$ ). Однако, как мы увидим ниже, вариация символа $(2.6)$ в слоях компактна, если все его символы $\sigma_{j}\left(\sigma_{0}(D)\right)$ равны нулю, $j=1, \ldots, k$.

ОПРЕДЕЛЕНИЕ 2.7. Конормалъным символом $\sigma_{c}\left(\sigma_{0}(D)\right) \in \Psi\left(\mathfrak{M}_{0}^{\circ} \times V \times \mathbb{R}, \Omega\right)$ семейства (2.6) называется семейство $\sigma_{c}\left(\sigma_{0}(D)\right)=P(x, 0, v, 0, p)$.

Условия согласования. Мы определили понятие ПДО с параметрами на стратифицированном многообразии $\mathfrak{M}$. Такие ПДО имеют символы, определенные априори на кокасательных расслоениях к открытым стратам, умноженных на пространство параметров без нулевого сечения. Однако, сравнивая представление (2.5) с представлением, имеющим место в $U \backslash \mathfrak{M}_{0}$ по индуктивному предположению, можно убедиться в том, что на самом деле эти символы непрерывно (и гладко) продолжаются на границу кокасательного расслоения и, тем самым, определены на кокасательных расслоениях к соответствующим замкнутым стратам. Более того, в точках примыкания страта $\mathfrak{M}_{j}$ к страту $\mathfrak{M}_{i}, j>i$, выполнены условия согласования:

$$
\left.\sigma_{l}\left(\sigma_{j}(D)\right)\right|_{\mathfrak{M}_{i}}=\sigma_{l}\left(\sigma_{i}(D)\right), \quad l=j, \ldots, k .
$$

Разумеется, здесь мы полагаем для краткости записи, что $\sigma_{j}\left(\sigma_{j}(D)\right)=\sigma_{j}(D)$. 
Основные свойства исчисления ПДО. Обозначим через $\Psi\left(T^{*} X \times V, K_{\Omega}\right)$ множество всевозможных символов вида (2.6) над многообразием $X=\mathfrak{M}_{0}$.

ПРЕДЛОЖЕНИЕ 2.1. Множество $\Psi\left(T^{*} X \times V, K_{\Omega}\right)$ является локальной $C^{*}$-алгеброй.

В качестве нормы можно взять супремум операторной нормы по всем значениям параметров.

ТЕОрема 2.1 (основные свойства ПДО). Псевдодифференииалънъе операторы обладают следующими свойствами:

1) множество $\Psi(V, \mathfrak{M})$ псевдодифференииальных операторов на стратифицированном многообразии $\mathfrak{M}$ является алгеброй относительно обычного умножения операторов и представляет собой локальную $C^{*}$-алгебру относительно супремума операторной нормы по параметру; псевдодифференииалъные операторы компактно коммутируют с операторами умножения на непрерывные функиии, заданнъе на $\mathfrak{M}$;

2) символьное отображение

$$
\begin{aligned}
\sigma: \Psi(V, \mathfrak{M}) & \rightarrow \bigoplus_{j=0}^{k} \Psi\left(T^{*} \mathfrak{M}_{j} \times V, K_{\Omega_{j}}\right), \\
D & \mapsto\left(\sigma_{0}(D), \ldots, \sigma_{k}(D)\right)
\end{aligned}
$$

является гомоморбизмом локальных $C^{*}$-алгебр и порождает изоморбизм

$$
\sigma: \Psi(V, \mathfrak{M}) / J(V, \mathfrak{M}) \rightarrow \Sigma(V, \mathfrak{M}) \subset \bigoplus_{j=0}^{k} \Psi\left(T^{*} \mathfrak{M}_{j} \times V, K_{\Omega_{j}}\right)
$$

на локальную $C^{*}$-алгебру символов, удовлетворяющих условиям согласования $(2.10)$; здесъ $J(V, \mathfrak{M}) \subset \Psi(V, \mathfrak{M})$ - идеал, состоящий из компактнозначньх операторных семейств, убывающих на бесконечности;

3) алгебра $\Psi(V, \mathfrak{M})$ инвариантна относительно дифбеоморбизмов многообразия $\mathfrak{M}$.

ОПРЕДЕЛЕНИЕ 2.8. Оператор $D \in \Psi(V, \mathfrak{M})$ называется эллиптическим, если все его символы $\sigma_{j}(D), j=0,1, \ldots, k$, обратимы вне нулевых сечений соответствующих расслоений.

Из теоремы 2.1 следует

Теорема 2.2. 1) На компактном стратифицированном многообразии М эллиптические операторы фредгольмовы (при каждом значении параметра).

2) Если $V \neq\{0\}$, то эллиптический с параметром оператор обратим при больиих $|v|$.

Далее нам будет также полезно следующее утверждение.

ПреДЛОЖенИЕ 2.2. Пусть $\Sigma_{0} \subset \Psi\left(V, K_{\Omega}\right)$ - множество символов, все символы которых, отвечающие стратам многообразия $\Omega \times \mathbb{R}_{+}$, равны нулю. Тогда произвольный элемент $\sigma \in \Sigma_{0}$ имеет компактную вариачию по переменной $v$. 
ДокАзАтЕльство. Согласно определению (см. выражение (2.6)) символ $\sigma$ имеет вид

$$
\sigma(v)=P\left(r v, i r \frac{\partial}{\partial r}+i \frac{n+1}{2}\right): L^{2}\left(K_{\Omega}\right) \rightarrow L^{2}\left(K_{\Omega}\right)
$$

для оператор-функции $P(w, p) \in \Psi(V \times \mathbb{R}, \Omega)$. В силу теоремы 2.1 выполнено условие $P(w, p) \in J(V \times \mathbb{R}, \Omega)$ и $P(w, p)$ имеет оценки типа (2.7) по переменным $(w, p)$.

Надо показать, что при $v \neq 0$ производная $\partial \sigma / \partial v$ является компактной. Имеем

$$
\frac{\partial \sigma}{\partial v}=r \frac{\partial P}{\partial w}\left(r v, i r \frac{\partial}{\partial r}+i \frac{n+1}{2}\right) .
$$

Символ $r \partial P / \partial w(r v, p)$ удовлетворяет всем необходимым оценкам для определения ограниченного оператора в $L^{2}\left(K_{\Omega}\right)$ (ср. (2.9)). Кроме того, он компактнозначен. Осталось показать, что он стремится к нулю при $r \rightarrow 0$ и при $r \rightarrow \infty$. При $r \rightarrow 0$ это очевидно (в силу наличия сомножителя $r$ ), а при $r \rightarrow \infty$ необходимо рассмотреть представление

$$
r \frac{\partial P}{\partial w}(r v, p)=\frac{1}{|v|}|r v| \frac{\partial P}{\partial w}(r v, p)=\frac{1}{|v|}\left[|w| \frac{\partial P}{\partial w}\right]_{w=r v}
$$

и использовать для функции $P(w, p)$ следующую лемму.

Лемма 2.1. Если $f \in C^{2}\left(\mathbb{R}^{n}\right)$ - оператор-функция такая, что $f(\xi) \rightarrow 0$ при $\xi \rightarrow \infty u\left|f^{\prime \prime}(\xi)\right| \leqslant C|\xi|^{-2}$ при больших $\xi$, mо

$$
|\xi|\left|f^{\prime}(\xi)\right| \rightarrow 0 \quad \text { npu } \quad \xi \rightarrow \infty .
$$

Квазиклассическое квантование. Квантование (2.6) можно рассматривать с "квазиклассическим" параметром $h$ :

$$
T_{h}: \Psi(V \times \mathbb{R}, \Omega) \rightarrow \Psi\left(V, K_{\Omega}\right) \subset \mathcal{B}\left(L^{2}\left(K_{\Omega}\right)\right) .
$$

При этом семейству с параметрами операторов на базе конуса сопоставляется семейство операторов на бесконечном конусе по следующему правилу:

$$
\left(T_{h} D\right)(v):=D\left(\stackrel{2}{r} v, i h r \frac{\frac{\partial}{\partial}}{\partial r}+i h \frac{n+1}{2}\right) .
$$

Так же, как в [8, приложение], можно показать, что это квантование является асимптотическим в $L^{2}$, т. е. удовлетворяет оценкам

$$
T_{h}(a) T_{h}(b)=T_{h}(a b)+o(1), \quad h \rightarrow 0,
$$

в смысле операторной нормы.

Мы используем такое квантование в дальнейшем при вычислении граничного отображения в $K$-теории алгебр ПДО. 


\section{§ 3. Ell-группы}

Через $\mathfrak{M}$, как и выше, будем обозначать стратифицированное многообразие. Всюду далее предполагается, что М компактно.

ОПРЕДЕЛЕНИЕ 3.1. Два эллиптических оператора

$$
D: L^{2}(\mathfrak{M}, E) \rightarrow L^{2}(\mathfrak{M}, F), \quad D^{\prime}: L^{2}\left(\mathfrak{M}, E^{\prime}\right) \rightarrow L^{2}\left(\mathfrak{M}, F^{\prime}\right),
$$

действующие в сечениях векторных расслоений на раздутии $M$ многообразия $\mathfrak{M}$, называются стабильно гомотопными, если существует непрерывная гомотопия ${ }^{4}$ эллиптических операторов

$$
D \oplus 1_{E_{0}} \sim f^{*}\left(D^{\prime} \oplus 1_{F_{0}}\right) e^{*}
$$

где $E_{0}, F_{0} \in \operatorname{Vect}(M)$ - векторные расслоения, а

$$
e: E \oplus E_{0} \rightarrow E^{\prime} \oplus F_{0}, \quad f: F^{\prime} \oplus F_{0} \rightarrow F \oplus E_{0}
$$

- изоморфизмы расслоений.

Здесь под эллиптичностью понимаем обратимость всех компонент символа оператора на стратах (см. определение 2.8) и рассматриваем только гомотопии псевдодифференциальных операторов, сохраняющие эллиптичность.

3.1. Четные группы $\operatorname{Ell}_{0}(\mathfrak{M})$. Стабильная гомотопность - отношение эквивалентности на множестве всех эллиптических псевдодифференциальных операторов, действующих в сечениях расслоений. Через $\operatorname{El}_{0}(\mathfrak{M})$ обозначим фактормножество по этому отношению эквивалентности. Это фактормножество является группой относительно прямой суммы эллиптических операторов. Обратный элемент определяется почти обратным оператором (т. е. обратным с точностью до компактных операторов).

3.2. Нечетные группы $\mathrm{Ell}_{1}(\mathfrak{M})$. Аналогичным образом определяется нечетная эллиптическая теория $\operatorname{Ell}_{1}(\mathfrak{M})$ как группа стабильных гомотопических классов эллиптических самосопряженных операторов. При этом стабилизация производится в терминах операторов $\pm \mathrm{Id}$.

ЗАмЕчаниЕ 3.1. Эквивалентное определение нечетной Ell-группы можно дать в терминах гладких семейств операторов на $\mathfrak{M}$, параметризованных окружностью $\mathbb{S}^{1}$, по модулю постоянных семейств.

Задача о гомотопической классификации эллиптических операторов состоит в том, чтобы вычислить группы $\mathrm{Ell}_{*}(\mathfrak{M})$.

\footnotetext{
${ }^{4}$ Всегда можно предполагать (это мы и будем делать далее), что рассматриваемые гомотопии таковы, что все символы вместе с производными гомотопируются непрерывно в смысле соответствующих норм.
} 


\section{§4. Основная теорема}

4.1. Отображение в $K$-гомологии. Пусть

$$
D: L^{2}(\mathfrak{M}, E) \rightarrow L^{2}(\mathfrak{M}, F)
$$

- эллиптический оператор, как в предыдущем параграфе. В силу теоремы 2.2 и теоремы $2.1,1)$ такой оператор может рассматриваться как обобщенный эллиптический оператор в смысле Атьи [1] на $\mathfrak{M}$ и поэтому определяет элемент в $K$-гомологиях многообразия $\mathfrak{M}$. Соответствующий фредгольмов модуль определяется стандартным образом. А именно (ср. [22]), если $D$ самосопряжен (и $E=F)$, будем рассматривать нормализацию

$$
\mathcal{D}=\left(P_{\text {ker } D}+D^{2}\right)^{-1 / 2} D: L^{2}(\mathfrak{M}, E) \rightarrow L^{2}(\mathfrak{M}, E),
$$

где $P_{\text {ker } D}$ - ортогональный проектор на ядро.

В общем случае будем рассматривать самосопряженный оператор

$$
\begin{gathered}
\mathcal{D}=\left(\begin{array}{cc}
0 & \left(P_{\text {ker } D}+D^{*} D\right)^{-1 / 2} D^{*} \\
D\left(P_{\text {ker } D}+D^{*} D\right)^{-1 / 2} & 0
\end{array}\right): \\
L^{2}(\mathfrak{M}, E \oplus F) \rightarrow L^{2}(\mathfrak{M}, E \oplus F),
\end{gathered}
$$

нечетный относительно $\mathbb{Z}_{2}$-градуировки пространства $L^{2}(\mathfrak{M}, E) \oplus L^{2}(\mathfrak{M}, F)$.

ПРЕДЛОЖЕНИЕ 4.1. 1) Операторь (4.1) u (4.2) определяют элементы 8 $K$-гомологиях, обозначаемые через $[D] \in K_{*}(\mathfrak{M})$, где * $=1$ для самосопряженных операторов $u *=0$ в общем случае.

2) Корректно определен гомоморфизм групп

$$
\mathrm{Ell}_{*}(\mathfrak{M}) \stackrel{\varphi}{\rightarrow} K_{*}(\mathfrak{M}), \quad D \mapsto[D] .
$$

ДокАЗАтЕльство. Операторы $\mathcal{D}$ из (4.1), (4.2) являются самосопряженными операторами, действующими в *-модуле над $C^{*}$-алгеброй $C(\mathfrak{M})$. Для завершения доказательства утверждения 1) предложения достаточно проверить, что для любой функции $f \in C(\mathfrak{M})$ имеют место соотношения

$$
[\mathcal{D}, f] \in \mathcal{K}, \quad\left(\mathcal{D}^{2}-1\right) f \in \mathcal{K},
$$

где $\mathcal{K}$ - идеал компактных операторов. Эти соотношения легко следуют из формулы композиции псевдодифференциальных операторов (оператор $\mathcal{D}$ является псевдодифференциальным). Отображение корректно определено, так как гомотопии эллиптических операторов дают непрерывные гомотопии соответствующих фредгольмовых модулей, т.е. приводят к тому же элементу в $K$-гомологиях. Изоморфизмы расслоений дают вырожденные фредгольмовы модули (напомним [22], что вырожденными называются модули, для которых выражения в левых частях (4.3) обращаются в нуль).

4.2. Классификационная теорема. Следующая теорема решает задачу о классификации для стратифицированных многообразий.

Теорема 4.1. Имеет место изоморфизм

$$
\operatorname{Ell}_{*}(\mathfrak{M}) \stackrel{\varphi}{\simeq} K_{*}(\mathfrak{M}),
$$

при котором эллиптическому оператору D сопоставляется элемент, определенный в предложении 4.1. 
Из невырожденности индексного спаривания $K_{0}(\mathfrak{M}) \times K^{0}(\mathfrak{M}) \rightarrow \mathbb{Z}$ (на свободных частях групп) получаем

СлеДСТВИЕ 4.1. Два эллиптических оператора $D_{1}$ u $D_{2}$ являются стабильно рационально гомотопными тогда и только тогда, когда равны их индексы с коэфбиииентами в произвольном векторном расслоении на $\mathfrak{M}$.

Теорему 4.1 мы получим как частный случай более общей теоремы, которую сформулируем ниже.

4.3. Классификация частично эллиптических операторов. Оператор $D$ на многообразии $\mathfrak{M}$ будем называть эллиптическим на множестве $\mathfrak{M} \backslash \mathfrak{M}_{j}$, если компоненты $\sigma_{k}(D), \ldots, \sigma_{j+1}(D)$ его символа являются обратимыми всюду на области своего определения, т. е. всюду вне нулевых сечений расслоений $T^{*} \mathfrak{M}_{k}, \ldots, T^{*} \mathfrak{M}_{j+1}$.

Через $\operatorname{Ell}_{*}\left(\mathfrak{M}, \mathfrak{M}_{j}\right)$ обозначим группу, состоящую из стабильных гомотопических классов псевдодифференциальных операторов, эллиптических на $\mathfrak{M} \backslash \mathfrak{M}_{j}$. Здесь подразумевается, что гомотопии рассматриваются в указанном классе операторов.

Аналогично отображению $\varphi$, построенному в предложении 4.1, определяется отображение

$$
\mathrm{Ell}_{*}\left(\mathfrak{M}, \mathfrak{M}_{j}\right) \stackrel{\varphi}{\rightarrow} K_{*}\left(\mathfrak{M} \backslash \mathfrak{M}_{j}\right)
$$

При этом необходимо вместо операторов $\left(P_{\operatorname{ker} D}+D^{*} D\right)^{-1 / 2}$ брать такие самосопряженные псевдодифференциальные операторы, старшие $k-j$ компонент символа которых есть

$$
\left(\sigma_{k}(D)^{*} \sigma_{k}(D)\right)^{-1 / 2}, \ldots,\left(\sigma_{j+1}(D)^{*} \sigma_{j+1}(D)\right)^{-1 / 2} .
$$

Заметим, что в случае оператора, эллиптического на всем многообразии $\mathfrak{M}$, обе конструкции дают один и тот же элемент $K$-гомологий.

ТеОРема 4.2. Для любого $-1 \leqslant j \leqslant k-1$ имеет место изоморфизм

$$
\operatorname{Ell}_{*}\left(\mathfrak{M}, \mathfrak{M}_{j}\right) \stackrel{\varphi}{\simeq} K_{*}\left(\mathfrak{M} \backslash \mathfrak{M}_{j}\right) .
$$

Доказательство теоремы 4.2 проведено в 5 5-7. Сначала, в $\S 5$, Ell-группы представляются как $K$-группы алгебр (некоммутативный аналог разностной конструкции Атьи-Зингера). Это позволяет определить точные последовательности для Ell-групп. Затем доказательство теоремы проводится индукцией по стратам в $\S 6$ и $\S 7$.

\section{§ 5. Связь Ell-групп и $K$-теории}

5.1. Ell-группы как $K$-группы $C^{*}$-алгебр. Псевдодифференциальные операторы в сечениях расслоений можно описать в терминах вложения алгебр

$$
C^{\infty}(M) \subset \Psi(\mathfrak{M})
$$

скалярных операторов (вложение отвечает обычному действию функций как операторов умножения). А именно, произвольный ПДО нулевого порядка в сечениях расслоений можно представить в виде

$$
D^{\prime}: \operatorname{Im} P \rightarrow \operatorname{Im} Q,
$$


где $P, Q$ - матричные проекторы $\left(P^{2}=P, Q^{2}=Q\right)$ с коэффициентами в алгебре функций $C^{\infty}(M)$, а $D^{\prime}$ - матричный оператор, компоненты которого лежат в операторной алгебре $\Psi(\mathfrak{M})$.

Через

$$
\Sigma\left(\mathfrak{M} \backslash \mathfrak{M}_{j}\right) \stackrel{\text { def }}{=} \operatorname{Im}\left(\sigma_{k}, \ldots, \sigma_{j+1}\right) \subset \bigoplus_{l \geqslant j+1} C^{\infty}\left(S^{*} M_{l}, \mathcal{B}\left(L^{2}\left(K_{\Omega_{l}}\right)\right)\right)
$$

обозначим алгебру, порожденную старшими $k-j$ компонентами символа.

В [5, теорема 4] доказаны изоморфизмы

$$
\operatorname{Ell}_{*}\left(\mathfrak{M}, \mathfrak{M}_{j}\right) \stackrel{\chi}{\simeq} K_{*}\left(\operatorname{Con}\left(C^{\infty}(M) \stackrel{f}{\rightarrow} \Sigma\left(\mathfrak{M} \backslash \mathfrak{M}_{j}\right)\right)\right)
$$

Ell-группы и $K$-группы специальной локальной $C^{*}$-алгебры. Здесь через

$$
f: C^{\infty}(M) \rightarrow \Sigma\left(\mathfrak{M} \backslash \mathfrak{M}_{j}\right)
$$

обозначено вложение, при котором гладкой функции на раздутии $M$ сопоставляется символ оператора умножения на эту функцию, а через

$$
\operatorname{Con}(A \stackrel{f}{\rightarrow} B)=\left\{(a, b(t)) \in A \oplus C_{0}([0,1), B) \mid f(a)=b(0)\right\}
$$

обозначен конус гомоморфизма алгебр $f: A \rightarrow B$.

В большинстве интересных случаев правую часть формулы (5.1) можно представить в эквивалентном виде, не содержащем конус отображения.

ЛЕмма 5.1. Имеем

$$
K_{*+1}\left(\operatorname{Con}\left(C^{\infty}(M) \rightarrow \Sigma\left(\mathfrak{M} \backslash \mathfrak{M}_{j}\right)\right)\right) \simeq K_{*}\left(\Sigma\left(\mathfrak{M} \backslash \mathfrak{M}_{j}\right)\right) / K_{*}\left(C^{\infty}(M)\right)
$$

при условии, что многообразие $M$ имеет ненулевое векторное поле (это условие выполнено, когда, например, $M$ не имеет замкнутых, т.е. без края, компонент).

ДокАЗАТЕЛЬство. Векторное поле $M \rightarrow S^{*} M$ определяет сечение $\Sigma(\mathfrak{M} \backslash$ $\left.\mathfrak{M}_{j}\right) \rightarrow C^{\infty}(M)$. Поэтому точная последовательность конуса вложения алгебр также расщепляется, что и дает искомый изоморфизм.

ЗАмЕчАНИЕ 5.1. В нечетном случае композиция последнего изоморфизма с гомоморфизмом $\chi$ показывает, что по модулю стабильных гомотопий эллиптические самосопряженные операторы изоморфны символам-проекторам по модулю проекторов, определяющих сечения расслоений (ср. с [23]).

5.2. Последовательность пары в Ell-теории. Построим точную последовательность в эллиптической теории, отвечающую паре пространств

$$
\mathfrak{M}_{j} \backslash \mathfrak{M}_{j-1} \subset \mathfrak{M} \backslash \mathfrak{M}_{j-1} .
$$

Отображения искомой последовательности мы определим в терминах отображений в $K$-теории. Для этого рассмотрим коммутативную диаграмму с точными строками

$$
\begin{aligned}
& 0 \rightarrow \operatorname{ker}\left(\sigma_{k}, \ldots, \sigma_{j+1}\right) \rightarrow \Sigma\left(\mathfrak{M} \backslash \mathfrak{M}_{j-1}\right) \stackrel{\left(\sigma_{k}, \ldots, \sigma_{j+1}\right)}{\longrightarrow} \Sigma\left(\mathfrak{M} \backslash \mathfrak{M}_{j}\right) \rightarrow 0
\end{aligned}
$$

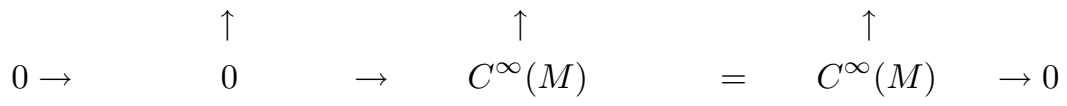


Идеал $\operatorname{ker}\left(\sigma_{k}, \ldots, \sigma_{j+1}\right)$, состоящий из символов с нулевыми старшими $k-j$ компонентами, обозначим для краткости через $\Sigma_{0}$. Диаграмма индуцирует точную последовательность

$$
0 \rightarrow S \Sigma_{0} \rightarrow \operatorname{Con}\left(C^{\infty}(M) \rightarrow \Sigma\left(\mathfrak{M} \backslash \mathfrak{M}_{j-1}\right)\right) \rightarrow \operatorname{Con}\left(C^{\infty}(M) \rightarrow \Sigma\left(\mathfrak{M} \backslash \mathfrak{M}_{j}\right)\right) \rightarrow 0
$$

конусов вертикальных вложений. Здесь $S \Sigma_{0}=C_{0}\left((0,1), \Sigma_{0}\right)$ - надстройка.

$K$-группы конусов в (5.3) классифицируют согласно (5.1) эллиптические операторы на многообразиях $\mathfrak{M} \backslash \mathfrak{M}_{j-1}$ и $\mathfrak{M} \backslash \mathfrak{M}_{j}$ соответственно. $K$-группы идеала также классифицируют эллиптические операторы.

ЛЕмма 5.2. K-группъ алгебры $S \Sigma_{0}$ классифицируют такие эллиптические на $\mathfrak{M} \backslash \mathfrak{M}_{j-1}$ операторьь, символь $\left(\sigma_{k}, \ldots, \sigma_{j+1}\right)$ которых индуцированы умножением на постоянные функиии из $C^{\infty}(M)$.

ДокАЗАТЕЛЬСтво. Для определенности рассмотрим случай четной группы $K_{0}\left(S \Sigma_{0}\right)$. Тогда $K_{0}\left(S \Sigma_{0}\right)=K_{1}\left(\Sigma_{0}\right)=K_{1}\left(\Sigma_{0}^{+}\right)$, где через $\Sigma_{0}^{+}$обозначена алгебра с добавленной единицей. Теперь из определения $K_{1}$-группы и идеала $\Sigma_{0}$ следует, что группа $K_{1}\left(\Sigma_{0}^{+}\right)$классифицирует эллиптические операторы, о которых идет речь в утверждении леммы.

В силу леммы 5.2 можно определить отображение (ср. с предложением 4.1)

$$
\varphi: K_{*}\left(S \Sigma_{0}\right) \rightarrow K_{*}\left(\mathfrak{M}_{j} \backslash \mathfrak{M}_{j-1}\right)
$$

с помощью перехода к сужению эллиптических операторов на окрестность $U$ подмножества $\mathfrak{M}_{j} \backslash \mathfrak{M}_{j-1}$, являющуюся расслоением $\pi: U \rightarrow \mathfrak{M}_{j} \backslash \mathfrak{M}_{j-1}$ со слоем конус. Отметим, что структура $C_{0}\left(\mathfrak{M}_{j} \backslash \mathfrak{M}_{j-1}\right)$-модуля

$$
C_{0}\left(\mathfrak{M}_{j} \backslash \mathfrak{M}_{j-1}\right) \stackrel{\pi^{*}}{\rightarrow} C(U) \rightarrow \mathcal{B}\left(L^{2}(U)\right)
$$

на соответствующих $L^{2}$-пространствах, в которых действует оператор, получается поднятием с базы расслоения, а сужение оператора корректно определено в силу того, что вне произвольно малой окрестности страта $\mathfrak{M}_{j} \backslash \mathfrak{M}_{j-1}$ оператор по построению является оператором умножения на функцию.

Ниже (см. лемму 7.1) мы покажем, что имеет место изоморфизм

$$
K_{*}\left(S \Sigma_{0}\right) \simeq K_{c}^{*}\left(T^{*} M_{j}\right)=\operatorname{Ell}_{*}\left(\mathfrak{M}_{j}, \mathfrak{M}_{j-1}\right) .
$$

Поэтому в последовательности, которую в $K$-теории индуцирует последовательность (5.3), можно заменить все $K$-группы на Ell-группы. В результате получаем периодическую шестичленную последовательность, связывающую Ell-группы:

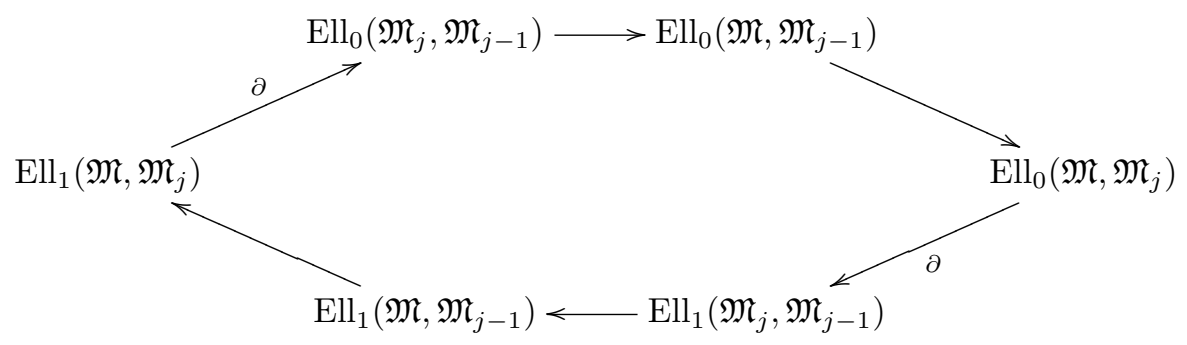


Здесь мы не рассматриваем вопрос об определении отображений этой последовательности непосредственно в терминах эллиптических операторов.

\section{§ 6. Индукция}

При индексе $j$, принимающем значения от $k$ до -1 , мы докажем по индукции, что отображение $\varphi$ из теоремы 4.2 является изоморфизмом на множестве $\mathfrak{M} \backslash \mathfrak{M}_{j}$.

При $j=k$ утверждение очевидно. Докажем индуктивный переход: из изоморфизма при $j$ следует изоморфизм при $j-1$.

Пара $\mathfrak{M}_{j} \backslash \mathfrak{M}_{j-1} \subset \mathfrak{M} \backslash \mathfrak{M}_{j-1}$ приводит к диаграмме

$$
\begin{aligned}
& \cdots \rightarrow \operatorname{Ell}_{*+1}\left(\mathfrak{M}, \mathfrak{M}_{j}\right) \stackrel{\partial}{\longrightarrow} \operatorname{Ell}_{*}\left(\mathfrak{M}_{j}, \mathfrak{M}_{j-1}\right) \rightarrow \operatorname{Ell}_{*}\left(\mathfrak{M}, \mathfrak{M}_{j-1}\right) \rightarrow \operatorname{Ell}_{*}\left(\mathfrak{M}, \mathfrak{M}_{j}\right) \stackrel{\partial}{\longrightarrow} \cdots \\
& \cdots \rightarrow K_{*+1}\left(\mathfrak{M} \backslash \mathfrak{M}_{j}\right) \stackrel{\partial}{\longrightarrow} K_{*}\left(\mathfrak{M}_{j} \backslash \mathfrak{M}_{j-1}\right) \rightarrow K_{*}\left(\mathfrak{M} \backslash \mathfrak{M}_{j-1}\right) \rightarrow K_{*}\left(\mathfrak{M} \backslash \mathfrak{M}_{j}\right) \stackrel{\partial}{\longrightarrow} \cdots
\end{aligned}
$$

Отметим, что вертикальные отображения диаграммы определены в силу предложения 4.1 и формул (5.4), (5.5). Если мы установим коммутативность диаграммы, то искомая изоморфность вертикального отображения со значениями в группе $K_{*}\left(\mathfrak{M} \backslash \mathfrak{M}_{j-1}\right)$ будет следовать из индуктивного предположения в силу леммы о пяти гомоморфизмах. Установим коммутативность.

Коммутативность квадрата

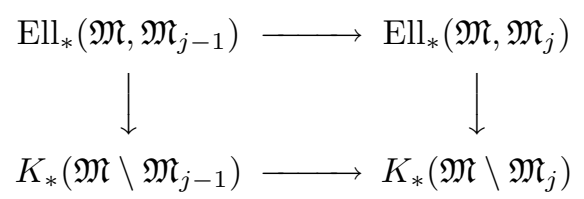

очевидна, так как отображения, действующие по горизонтали, отвечают "забыванию" соответствующих структур.

Коммутативность квадрата

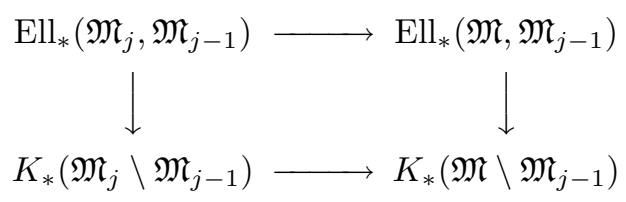

отвечающего вложению $i: \mathfrak{M}_{j} \backslash \mathfrak{M}_{j-1} \rightarrow \mathfrak{M} \backslash \mathfrak{M}_{j-1}$, также несложно установить. В самом деле, если мы рассмотрим композицию отображений, проходящую через правый верхний угол квадрата (6.2), то она сопоставляет эллиптическому оператору на $\mathfrak{M} \backslash \mathfrak{M}_{j-1}$, равному оператору умножения вне окрестности $U$ страта $\mathfrak{M}_{j} \backslash \mathfrak{M}_{j-1}$, этот же оператор с естественной структурой $C_{0}\left(\mathfrak{M} \backslash \mathfrak{M}_{j-1}\right)$-модуля на пространствах, в которых он действует. Если теперь сузить оператор на окрестность страта $\mathfrak{M}_{j} \backslash \mathfrak{M}_{j-1}$ (элемент в $K$-гомологиях при этом не изменится, так как соответствующий фредгольмов модуль изменится на вырожденный модуль), а затем модульную структуру гомотопией свести к композиции

$$
C_{0}\left(\mathfrak{M} \backslash \mathfrak{M}_{j-1}\right) \stackrel{i^{*}}{\longrightarrow} C_{0}\left(\mathfrak{M}_{j} \backslash \mathfrak{M}_{j-1}\right) \stackrel{\pi^{*}}{\longrightarrow} C(U) \longrightarrow \mathcal{B}\left(L^{2}(U)\right),
$$


где $\pi: U \rightarrow \mathfrak{M}_{j} \backslash \mathfrak{M}_{j-1}$ - проекция, то получится элемент, который сопоставлялся бы оператору, если рассмотреть композицию отображений, проходящую через левый нижний угол квадрата. Коммутативность квадрата (6.2) установлена.

Для обоснования индуктивного перехода осталось только проверить коммутативность квадратов, содержащих граничные отображения диаграммы (6.1). Эта проверка представляет собой самый технически сложный момент доказательства, и мы ее сделаем в следующем параграфе.

\section{§ 7. Сравнение граничных отображений}

В этом параграфе мы докажем коммутативность квадратов диаграммы (6.1), которые содержат граничные отображения. Соответствующие вычисления будем производить в терминах $K$-групп алгебр символов. Так как доказательство является достаточно длинным, то приведем сначала его схему:

(i) покажем, что граничное отображение в $K$-гомологиях является композицией сужения на границу страта и прямого образа (см. п. 7.1 ниже);

(ii) граничное отображение в $K$-теории также является композицией сужения и некоторого граничного отображения $\partial^{\prime}$, отвечающего алгебре $\Psi\left(T^{*} M_{j}, K_{\Omega}\right)$ символов на страте $M_{j}$ (п. 7.2), поэтому, чтобы сравнить граничные отображения в $K$-теории и в $K$-гомологиях, произведем вычисление на страте $M_{j}$;

(iii) непосредственно работать с граничным отображением $\partial^{\prime}$ достаточно трудно, поэтому заменим алгебру $\Psi\left(T^{*} M_{j}, K_{\Omega}\right)$ на алгебру $\Psi\left(T^{*} M_{j} \times \mathbb{R}, \Omega\right)$ семейств с параметрами, граничное отображение $\partial^{\prime \prime}$ для которой проще описать; более точно, в п. 7.3 определим асимптотический гомоморфизм, действующий из одной алгебры в другую, а в п. 7.4 покажем, что асимптотический гомоморфизм индуцирует изоморфизм $K$-групп;

(iv) граничное отображение $\partial^{\prime \prime}$, которое отвечает алгебре $\Psi\left(T^{*} M_{j} \times \mathbb{R}, \Omega\right)$, выражается в п. 7.5 в терминах индекса семейств эллиптических операторов с параметрами, после этого согласованность граничного отображения $\partial^{\prime \prime}$ с прямым образом в $K$-гомологиях и, как следствие, искомую коммутативность диаграммы (6.1) получим в п. 7.5.

Перейдем к подробному доказательству коммутативности диаграммы (6.1).

7.1. Граничное отображение в нижней строке диаграммы (6.1). Зафиксируем некоторые обозначения. Пусть $U$ - окрестность открытого страта $\mathfrak{M}_{j} \backslash \mathfrak{M}_{j-1}=\mathfrak{M}_{j}^{\circ}$, которая расслаивается над этим стратом со слоем конус. Через $\pi: U \rightarrow \mathfrak{M}_{j} \backslash \mathfrak{M}_{j-1}$ обозначим проекцию. Расслоение соответствующих баз конусов обозначим через

$$
\pi^{\prime}: \Xi \rightarrow \mathfrak{M}_{j} \backslash \mathfrak{M}_{j-1}
$$

Типичный слой расслоения обозначим через $\Omega$. Очевидно, имеет место соотношение $U \backslash \mathfrak{M}_{j}^{\circ} \simeq \mathbb{R}_{+} \times \Xi$. Тогда граничное отображение $\partial: K_{*}\left(\mathfrak{M} \backslash \mathfrak{M}_{j}\right) \rightarrow$ $K_{*+1}\left(\mathfrak{M}_{j} \backslash \mathfrak{M}_{j-1}\right)$ совпадает с композицией

$$
K_{*}\left(\mathfrak{M} \backslash \mathfrak{M}_{j}\right) \longrightarrow K_{*}\left(U \backslash \mathfrak{M}_{j}^{\circ}\right)=K_{*+1}(\Xi) \stackrel{\pi_{*}^{\prime}}{\longrightarrow} K_{*+1}\left(\mathfrak{M}_{j} \backslash \mathfrak{M}_{j-1}\right)
$$


сужения на окрестность $U \backslash \mathfrak{M}_{j}^{\circ}$, изоморфизма периодичности и прямого образа. Такое разложение несложно доказать, пользуясь естественностью граничного отображения.

7.2. Редукция на границу. Вычислим граничное отображение в $K$-теории алгебр. Из естественности граничного отображения

$$
\partial: K_{*}\left(\operatorname{Con}\left(C^{\infty}(M) \rightarrow \Sigma\left(\mathfrak{M} \backslash \mathfrak{M}_{j}\right)\right)\right) \rightarrow K_{*+1}\left(S \Sigma_{0}\right)
$$

следует, что оно есть композиция

$$
\begin{aligned}
& K_{*}\left(\operatorname{Con}\left(C^{\infty}(M) \rightarrow \Sigma\left(\mathfrak{M} \backslash \mathfrak{M}_{j}\right)\right)\right) \\
& \quad \longrightarrow K_{*}\left(\operatorname{Con}\left(C^{\infty}\left(\partial_{j} M\right) \rightarrow \Sigma_{M_{j}}\right)\right) \stackrel{\partial^{\prime}}{\longrightarrow} K_{*+1}\left(S \Sigma_{0}\right)
\end{aligned}
$$

с сужением символов на страт $M_{j}$, где $\Sigma_{M_{j}} \equiv \Sigma\left(T^{*} M_{j}, \Omega\right)$ - алгебра символов псевдодифференциальных операторов на $\Omega$ с параметрами в $T^{*} M_{j}$ (здесь и далее мы фиксируем некоторый изоморфизм $\left.T^{*} \mathfrak{M}_{j} \simeq T^{*} M_{j}\right)$, а через $\partial_{j} M \subset \partial M$ обозначаем замыкание множества $\pi^{-1} \mathfrak{M}_{j}^{\circ}$. Заметим, что $\partial_{j} M-$ многообразие, которое расслаивается над $M_{j}$ со слоем, изоморфным раздутию стратифицированного многообразия $\Omega$.

7.3. Асимптотический гомоморфизм. Напомним, что $\Psi\left(T^{*} M_{j}, K_{\Omega}\right)-$ алгебра, образованная $j$-ми символами на страте $M_{j}$. Для вычисления граничного отображения $\partial^{\prime}$ мы заменим алгебру $\Psi\left(T^{*} M_{j}, K_{\Omega}\right)$ (не меняя ее $K$-группы) на алгебру псевдодифференциальных операторов с параметрами, для которой граничное отображение устроено проще.

Для этого определим отображение (см. (2.12))

$$
\begin{gathered}
T_{h}: \Psi\left(T^{*} M_{j} \times \mathbb{R}, \Omega\right) \longrightarrow \Psi\left(T^{*} M_{j}, K_{\Omega}\right), \quad h \in(0,1], \\
\left(T_{h} u\right)(\xi)=u\left(\stackrel{2}{r} \xi, i h r \frac{\partial}{\partial r}+i h \frac{n+1}{2}\right), \quad(\xi, p) \in T^{*} M_{j} \times \mathbb{R},
\end{gathered}
$$

где $\Psi\left(T^{*} M_{j} \times \mathbb{R}, \Omega\right)$ - алгебра гладких семейств ПДО на слоях $\Omega$ с параметрами в $T^{*} M_{j} \times \mathbb{R}$. При $h \rightarrow 0$ выполнены соотношения

$$
T_{h}(a b)=T_{h}(a) T_{h}(b)+o(1), \quad\left(T_{h}(a)\right)^{*}=T_{h}\left(a^{*}\right)+o(1),
$$

где $a, b \in \Psi\left(T^{*} M_{j} \times \mathbb{R}, \Omega\right)$ - произвольные элементы, а $o(1)$ отвечает равномерной операторной норме.

Указанное квазиклассическое квантование является частным случаем так называемых асимптотических гомоморфизмов, играющих важную роль в теории $C^{*}$-алгебр [24]-[26]. В частности, из соотношений (7.3) следует, что квантование $T_{h}$ индуцирует гомоморфизм $K$-групп

$$
T: K_{*}\left(\Psi\left(T^{*} M_{j} \times \mathbb{R}, \Omega\right)\right) \rightarrow K_{*}\left(\Psi\left(T^{*} M_{j}, K_{\Omega}\right)\right) .
$$

Рассмотрим теперь коммутативную диаграмму

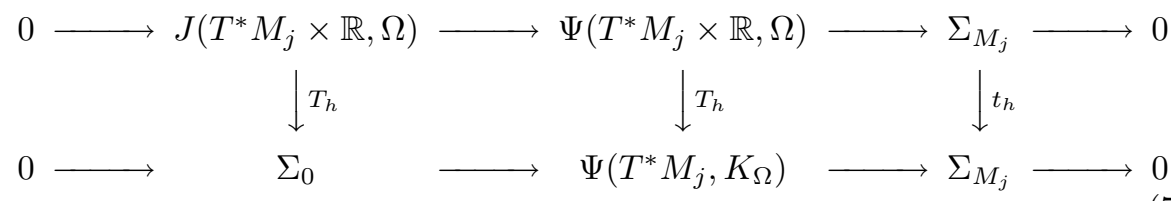


где $t_{h}$ - индуцированное отображение на символах семейств (оно является изоморфизмом алгебр).

Алгебра $C^{\infty}\left(\partial_{j} M\right)$ вложена в каждую из алгебр $\Psi\left(T^{*} M_{j} \times \mathbb{R}, \Omega\right)$, $\Psi\left(T^{*} M_{j}, K_{\Omega}\right)$ и $\Sigma_{M_{j}}$. Диаграмма, состоящая из конусов этих вложений, дает квадрат $K$-групп

$$
\begin{array}{ccc}
K_{*}\left(\operatorname{Con}\left(C^{\infty}\left(\partial_{j} M\right) \rightarrow \Sigma_{M_{j}}\right)\right) \stackrel{\partial^{\prime \prime}}{\longrightarrow} & K_{*}\left(C_{0}\left(T^{*} M_{j} \times \mathbb{R}\right)\right) \\
\| & \downarrow{ }^{T} \\
K_{*}\left(\operatorname{Con}\left(C^{\infty}\left(\partial_{j} M\right) \rightarrow \Sigma_{M_{j}}\right)\right) \stackrel{\partial^{\prime}}{\longrightarrow} & K_{*+1}\left(S \Sigma_{0}\right)
\end{array}
$$

(по горизонтали действуют граничные отображения соответствующих последовательностей). Здесь мы воспользовались изоморфизмом

$$
K_{*}\left(J\left(T^{*} M_{j} \times \mathbb{R}, \Omega\right)\right) \simeq K_{*}\left(C_{0}\left(T^{*} M_{j} \times \mathbb{R}\right)\right) .
$$

(Изоморфизм индуцирован вложением $J\left(T^{*} M_{j} \times \mathbb{R}, \Omega\right) \subset C_{0}\left(T^{*} M_{j} \times \mathbb{R}, \mathcal{K}\right)$ локальной $C^{*}$-алгебры в ее замыкание.)

Коммутативность квадрата (7.5) следует из естественности граничного отображения в $K$-теории по отношению к асимптотическим гомоморфизмам (см., например, [27]).

7.4. Отображение $T: K_{*}\left(C_{0}\left(T^{*} M_{j} \times \mathbb{R}\right)\right) \rightarrow K_{*}\left(\Sigma_{0}\right)$. Вычислим сначала группы $K_{*}\left(S \Sigma_{0}\right)$. Для этого рассмотрим короткую точную последовательность локальных $C^{*}$-алгебр

$$
0 \longrightarrow \operatorname{ker} \sigma_{c} \longrightarrow \Sigma_{0} \stackrel{\sigma_{c}}{\longrightarrow} J\left(M_{j} \times \mathbb{R}, \Omega\right) \longrightarrow 0
$$

( $\sigma_{c}$ - отображение, сопоставляющее оператору его конормальный символ; см. определение 2.7). Алгебра ker $\sigma_{c}$ состоит из семейств компактных операторов ${ }^{5}$. Так как вложения

$$
\operatorname{ker} \sigma_{c} \subset C\left(S^{*} M_{j}, \mathcal{K}\left(K_{\Omega}\right)\right), \quad J\left(M_{j} \times \mathbb{R}, \Omega\right) \subset C_{0}\left(M_{j} \times \mathbb{R}, \mathcal{K}(\Omega)\right)
$$

индуцируют изоморфизмы $K$-групп, то последовательность $K$-групп, отвечающую последовательности (7.6), можно записать как верхнюю строку диаграммы

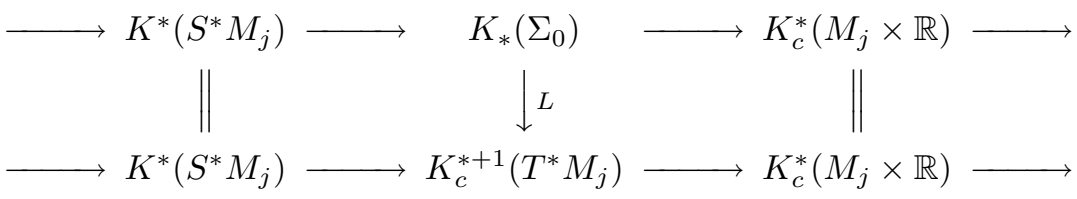

\footnotetext{
${ }^{5}$ Как и в случае многообразий с изолированными особенностями, семейство из $\Sigma_{0}$ компактно тогда и только тогда, когда его конормальный символ равен нулю.
} 
в которой нижняя строка - последовательность топологических $K$-групп пары $S^{*} M_{j} \subset B^{*} M_{j}$, образованной расслоениями единичных сфер и шаров в $T^{*} M_{j}$, а отображение $L$ - разностная конструкция для ПДО с операторнозначными символами по Люк (см. [20], [28]). Напомним, что это отображение определяется следующим образом: элемент $\sigma_{j}$ алгебры $\Sigma_{0}^{+}$с добавленной единицей является операторнозначной функцией на $S^{*} M_{j}$, обладающей компактной вариацией в слоях $S_{x}^{*} M_{j}$ (см. предложение 2.2 ); если эта функция обратима, то отображение $L$ на элементе $\left[\sigma_{j}\right] \in K_{1}\left(\Sigma_{0}\right)$ определяется как индекс

$$
L\left[\sigma_{j}\right]:=\operatorname{ind} \tilde{\sigma}_{j} \in K_{c}\left(T^{*} M_{j}\right)
$$

продолжения $\tilde{\sigma}_{j}$ функции $\sigma_{j}$ на расслоение единичных шаров в $T^{*} M_{j}$ с сохранением свойства компактной вариации в слоях (указанное продолжение является семейством, фредгольмовым на $B^{*} M_{j}$ и обратимым на $S^{*} M_{j}$, поэтому его индекс лежит в указанной $K$-группе с компактными носителями). Индекс (7.8) не зависит от продолжения. Аналогично определяется отображение $L$ в случае четных $K$-групп.

Лемма 7.1. Диаграмма (7.7) коммутативна, и, следовательно, $L$ - изоморфизм.

ДокАЗАТЕЛЬство. 1. Коммутативность квадратов

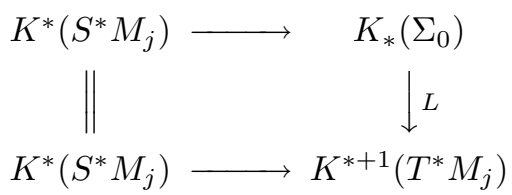

следует из того, что для конечномерных символов разностная конструкция $L$ совпадает с разностной конструкцией Атьи-Зингера.

2. Рассмотрим теперь квадрат

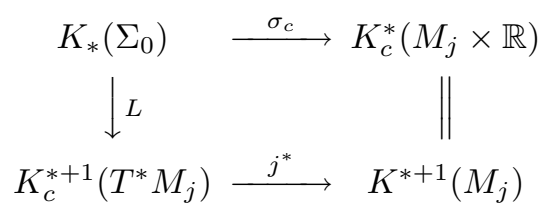

где $j: M_{j} \rightarrow T^{*} M_{j}$ - вложение нулевого сечения. Его коммутативность можно получить из формулы индекса (см., например, [29])

$$
\beta \text { ind } D_{y}=\operatorname{ind} \sigma_{c}\left(D_{y}\right) \in K_{c}^{1}(Y \times \mathbb{R})
$$

для семейства эллиптических операторов $D_{y}, y \in Y$, с единичным внутренним символом на бесконечном конусе. Через $Y$ обозначено компактное пространство параметров, а $\beta$ - изоморфизм периодичности $K(Y) \simeq K_{c}^{1}(Y \times \mathbb{R})$.

Действительно, в случае $a \in K_{1}\left(\Sigma_{0}\right)$ элементы $j^{*} L(a)$ и $\sigma_{c}(a)$ задают соответственно левую и правую части формулы индекса (7.9). В случае $a \in K_{0}\left(\Sigma_{0}\right)$ необходимо сначала перейти к надстройке, а затем применять формулу (7.9).

3. Рассмотрим теперь квадрат

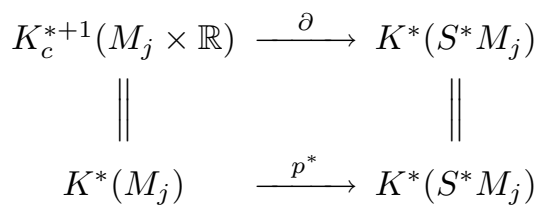


где $p: S^{*} M_{j} \rightarrow M_{j}$ - естественная проекция. Коммутативность этого квадрата также следует из формулы индекса (7.9). В самом деле, для элемента $a \in K_{c}^{1}\left(M_{j} \times \mathbb{R}\right)$, представимого семейством обратимых конормальных символов с единичным символом, проходя через левый нижний угол квадрата, мы получаем элемент $p^{*}$ ind $a \in K^{0}\left(S^{*} M_{j}\right)$. С другой стороны, проходя через правый верхний угол квадрата, получаем

$$
\partial a=p^{*} \text { ind } \hat{a},
$$

где $\hat{a}$ - семейство операторов на конусе $K_{\Omega}$ с внутренним символом, равным единице, и с конормальным символом, равным $а$. Отметим, что последнее соотношение вытекает из того факта, что граничное отображение $\partial$ сопоставляет обратимому символу индекс соответствующего оператора. Применяя формулу (7.9), получаем искомое соотношение $p^{*}$ ind $a=p^{*}$ ind $\hat{a} \in K^{0}\left(S^{*} M_{j}\right)$. В случае $a \in K_{c}^{0}\left(M_{j} \times \mathbb{R}\right)$ необходимо сначала перейти к надстройке.

ЛЕмма 7.2. Отображение

$$
T: K_{*}\left(C_{0}\left(T^{*} M_{j} \times \mathbb{R}\right)\right) \rightarrow K_{*}\left(\Sigma_{0}\right)
$$

является изоморфизмом, обратным к $L$ (см. (7.7)).

ДокАЗАтЕЛЬСтво. Рассмотрим, например, отображение

$$
T: K_{1}\left(C_{0}\left(T^{*} M_{j} \times \mathbb{R}\right)\right) \rightarrow K_{1}\left(\Sigma_{0}\right) .
$$

Докажем, что композиция $L T$ задает тождественное отображение на $K$-группе.

1. В самом деле, на символе $u(\xi, p) \in\left(1+J\left(T^{*} M_{j} \times \mathbb{R}, \Omega\right)\right)$, обратимом при $(\xi, p) \in T^{*} M_{j} \times \mathbb{R}$ и равном тождественно единице вне компакта, это отображение определяется формулой

$$
L T[u]=\operatorname{ind} u\left(\stackrel{2}{r} \xi, i h r \frac{1}{\partial r}+i h \frac{n+1}{2}\right) \in K_{c}^{0}\left(T^{*} M_{j}\right)
$$

(при достаточном малом $h),[u] \in K_{c}^{1}\left(T^{*} M_{j} \times \mathbb{R}\right)$. Индексный элемент определен корректно, так как операторнозначная функция $\left(T_{h} u\right)(\xi)$ имеет компактную послойную вариацию на $T^{*} M_{j} \backslash \mathbf{0}$ и обратима при $\xi \neq 0$ (см. предложение 2.2 и формулу (2.13) соответственно).

2. Через $\overline{T_{h} u}$ обозначим семейство фредгольмовых параметризованных $T^{*} M_{j} \backslash \mathbf{0}$ операторов, совпадающее при $|\xi|<1$ с семейством $T_{h} u$, а при $|\xi| \geqslant 1$ равное семейству

$$
u\left((\stackrel{2}{r}+|\xi|-1) \xi, i h r \frac{\frac{1}{\partial}}{\partial r}+i h \frac{n+1}{2}\right) .
$$

При достаточно малом $h$ это семейство будет обратимо при всех $\xi$ (это следует из ограниченности носителя разности $1-u$, а также из того, что оценки $(2.13)$ выполнены равномерно по параметру const $>\lambda \geqslant 0$, если в формуле $(2.12)$ заменить $r \xi$ на $(r+\lambda) \xi)$. По построению имеем

$$
\operatorname{ind} T_{h} u=\operatorname{ind} \overline{T_{h} u} \text {. }
$$


Однако семейство $\overline{T_{h} u}$ при $\xi$ вне компакта состоит из единичных операторов, поэтому его индекс вычисляется по формуле (7.9) и равен индексу семейства конормальных символов $u(\xi, p)$ (по модулю изоморфизма периодичности Ботта), т. е. действительно дает исходный элемент $K$-группы $L T[u]=[u]$.

7.5. Сравнение граничных отображений. Сравним полученные выражения для граничных отображений в $K$-гомологиях и в $K$-теории алгебр (см. (7.1) и $(7.2),(7.5))$. Отображения сужения из пп. 7.1, 7.2 выписывать не будем. Рассмотрим диаграмму (здесь и ниже $I=(0,1)$ )

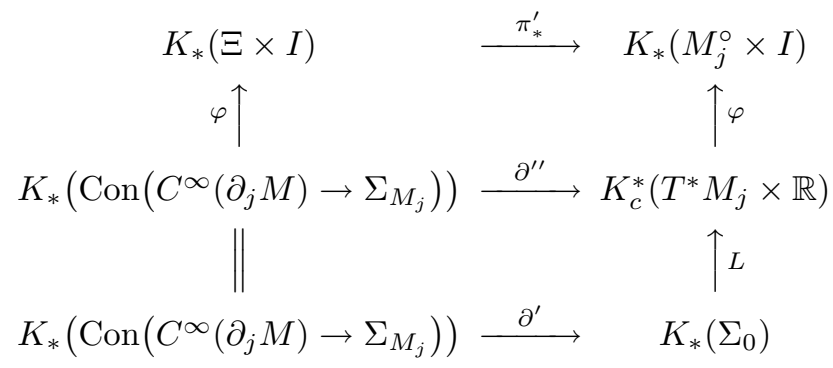

включающую граничные отображения в средней и нижней строках. Не определено пока только вертикальное отображение $\varphi$ в левом столбце. Чтобы его определить, заметим, что группы $K_{*}\left(\operatorname{Con}\left(C^{\infty}\left(\partial_{j} M\right) \rightarrow \Sigma_{M_{j}}\right)\right)$ классифицируют эллиптические семейства $\sigma(x, \xi, p)$ операторов на $\Omega$ с параметрами $(x, \xi, p) \in T^{*} M_{j} \times \mathbb{R}$. Такому семейству можно сопоставить оператор на произведении $\Xi \times I$ по формуле

$$
\sigma\left(x,-i \frac{\partial}{\partial x},-i \frac{\partial}{\partial t}\right) .
$$

Такой оператор определяет элемент группы $K_{*}(\Xi \times I)$ при условии эллиптичности семейства $\sigma(x, \xi, p)$.

Нижний квадрат диаграммы (7.10) изоморфен квадрату (7.5) в силу леммы 7.2. Поэтому он коммутативен. Мы утверждаем, что и верхний квадрат диаграммы коммутативен. Пусть $z \in K_{*}\left(\operatorname{Con}\left(C^{\infty}\left(\partial_{j} M\right) \rightarrow \Sigma_{M_{j}}\right)\right)$ - элемент, определяемый некоторым эллиптическим семейством $\sigma(x, \xi, p)$. Тогда из эллиптичности семейства следует его фредгольмовость и граничное отображение $\partial^{\prime \prime}$, примененное к элементу $z$, есть просто индекс семейства с параметрами в $T^{*} M_{j} \times \mathbb{R}$. С другой стороны, элемент $\pi_{*}^{\prime} z$ отвечает эллиптическому оператору

$$
\sigma\left(x,-i \frac{\partial}{\partial x},-i \frac{\partial}{\partial t}\right),
$$

рассматриваемому как оператор, действующий в $C_{0}\left(M_{j}^{\circ} \times I\right)$-модулях. Последние два элемента в действительности совпадают, что вытекает из следующего общего утверждения.

ТеОРема 7.1. Пусть $p(x, \xi)$ - операторнозначный символ на компактном многообразии с углами $X$, эллиптический по Люк [20]. Тогда имеет место равенство

$$
\left[p\left(x,-i \frac{\partial}{\partial x}\right)\right]=\varphi(\operatorname{ind} p(x, \xi)) \in K_{*}\left(X^{\circ}\right),
$$


где через $X^{\circ}$ обозначена внутренность многообразия, квадратные скобки обозначают элемент в $K$-гомологиях, а $\varphi: K_{c}^{*}\left(T^{*} X\right) \rightarrow K_{*}\left(X^{\circ}\right)-$ изоморбизм Пуанкаре на многообразии с углами (см., например, [30]).

Доказательство теоремы 7.1 приведено в дополнении (см. §9).

Итак, теорема 7.1 доставляет коммутативность диаграммы (7.10). Следовательно, коммутативны квадраты диаграммы (6.1), содержащие граничные отображения.

Доказательство теоремы 4.2 закончено.

\section{§ 8. Приложения}

8.1. Топологическое препятствие к фредгольмовости. Пусть $\mathfrak{M} \supset$ $X$ - стратифицированная пара. Интерес представляет вопрос о нахождении условий, при которых оператор, эллиптический на $\mathfrak{M} \backslash X$, можно модифицировать в эллиптический оператор на $\mathfrak{M}$, не меняя компонент символа на множестве $\mathfrak{M} \backslash X$. Этот вопрос аналогичен проблеме Атьи-Ботта о выяснении топологических условий, налагаемых на символ, при выполнении которых для оператора на многообразии с гладким краем существует фредгольмова краевая задача.

Мы ответим на аналогичный вопрос для элементов Ell-групп в случае произвольной стратификации. Для этого рассмотрим диаграмму

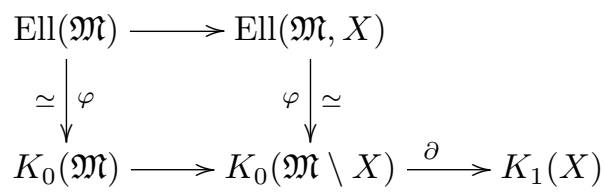

Ее коммутативность очевидна: рассматриваем отображение “забывания". Как следствие, получаем, что отличие от нуля элемента $\partial \varphi(a)$ является необходимым и достаточным условием для существования поднятия элемента $a \in$ $\operatorname{Ell}(\mathfrak{M}, X)$ в группу $\operatorname{Ell}(\mathfrak{M})$.

Граничное отображение в $K$-гомологиях играет аналогичную роль препятствия в других задачах (см. [31]-[33]).

Отметим, что равенство нулю элемента $\partial \varphi(a)$ является условием для внутреннего символа оператора (конечномерное условие), если $X=\mathfrak{M}_{k-1}$ - подмножество всех особенностей многообразия $\mathfrak{M}=\mathfrak{M}_{k}$.

8.2. Инвариантность индекса относительно кобордизма. Рассмотрим обобщение обычной инвариантности индекса оператора Дирака на гладком многообразии относительно кобордизмов. Предположим, что $X$ - гладкий страт. Тогда мы имеем коммутативную диаграмму

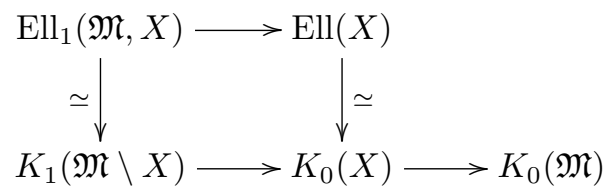


Поскольку отображение $K_{0}(X) \rightarrow K_{0}(\mathfrak{M})$ сохраняет индекс $(\in \mathbb{Z})$, то мы получаем, что индекс некоторого эллиптического оператора $D$ на $X$ будет равен нулю, если элемент $[D] \in \operatorname{Ell}(X)$ является образом элемента из $\operatorname{Ell}_{1}(\mathfrak{M}, X)$.

ЗАмЕчАниЕ 8.1. Для негладких $X$ построение такой коммутативной диаграммы - открытая задача (не известна уже соответствующая точная последовательность в Ell-теории).

\section{§ 9. Дополнение. Доказательство теоремы 7.1}

Отметим, что теорема 7.1 является уточнением теоремы Люк [20] об индексе операторов с операторнозначными символами (теорема об индексе получается из равенства (7.11), если считать, что $\partial X=\varnothing$, и применить индексное отображение к рассматриваемым элементам $K$-гомологий).

В случае однородных при больших $|\xi|$ символов приведенная формула доказывается так же, как в [20]. Общий случай (неоднородные символы) сводится к случаю однородных символов способом, схема которого была предложена в [28] и была приспособлена для вычисления индекса. Редукция, которую мы приводим в настоящей работе, основана на следующем стандартном факте $K K$-теории Каспарова.

ПРеДЛОЖениЕ 9.1. Пусть $P_{t}-$ *-сильно непрерывная гомотопия ограниченных операторов такая, что семейства

$$
f\left[P_{t} P_{t}^{*}-1\right], \quad f\left[P_{t}^{*} P_{t}-1\right], \quad\left[f, P_{t}\right], \quad\left[f, P_{t}^{*}\right] \quad \forall f \in C_{0}\left(X^{\circ}\right)
$$

являются непрерывными по норме семействами компактных операторов. Тогда соответствующий элемент в $K$-гомологиях не зависит от параметра:

$$
\left[P_{0}\right]=\left[P_{1}\right] \in K_{*}\left(X^{\circ}\right) .
$$

ДокАЗАТЕЛЬство. Условия предложения означают, что семейство $\left\{P_{t}\right\}$ определяет оператор в $\left(C_{0}\left(X^{\circ}\right), C([0,1])\right)$-бимодуле $C_{0}\left(X^{\circ} \times[0,1], L^{2}(H)\right)$. Этот оператор определяет гомотопию (в смысле $K K$-теории) между $P_{0}$ и $P_{1}$ (см., например, [34]). Поэтому независимость элемента $\left[P_{t}\right]$ в $K$-гомологиях от параметра $t$ вытекает из эквивалентности определения $K$-гомологий, использующего гомотопии, и определения, использующего операторные гомотопии.

Применим предложение 9.1 в рассматриваемой ситуации. Без ограничения общности можно считать, что символ $p(x, \xi)$ является гладким вплоть до нулевого сечения в $T^{*} X$ и нормализован: $p^{*}(x, \xi) p(x, \xi)=1$ при больших $|\xi|$. Пусть $\psi(t), t \geqslant 0,-$ такая гладкая положительная функция, что

$$
\psi(t)=\left\{\begin{array}{lll}
1 & \text { при } t<1 \\
1 / t & \text { при } \quad t>2 .
\end{array}\right.
$$

Рассмотрим символ

$$
p_{\varepsilon}(x, \xi)=p(x, \xi \psi(\varepsilon|\xi|)) .
$$

Непосредственным вычислением (ср. с [35, теорема 19.2.3]) устанавливаются следующие свойства: 
1) $p_{0}=p$; при $\varepsilon>0$ символ $p_{\varepsilon}$ является однородным при больших $|\xi|$, и при достаточно малых $\varepsilon$ он эллиптичен;

2) $p_{\varepsilon}$ и $p_{\varepsilon}^{*}$ равномерно ограничены в классе символов компактной вариации по слою на кокасательном расслоении $T^{*} X$ при $\varepsilon \in[0,1]$;

3) при достаточно малых $\varepsilon>0$ финитные компактнозначные символы $p_{\varepsilon} p_{\varepsilon}^{*}-1, p_{\varepsilon}^{*} p_{\varepsilon}-1$ не зависят от $\varepsilon$.

Определим оператор

$$
P_{\varepsilon}=p_{\varepsilon}\left(x,-i \frac{\partial}{\partial x}\right)
$$

(фиксируя на $X$ не зависящие от $\varepsilon$ координатные окрестности и подчиненное им разбиение единицы). Мы утверждаем, что (при достаточно малых $\varepsilon \geqslant 0$ ):

(a) ind $p_{\varepsilon} \in K_{c}\left(T^{*} X\right)$ не зависит от $\varepsilon$;

(b) семейство $P_{\varepsilon}$ удовлетворяет условиям предложения 9.1.

Отсюда будет вытекать теорема 7.1, поскольку, с одной стороны, при $\varepsilon>0$ символ $p_{\varepsilon}$ однороден на бесконечности, так что справедливо равенство $\left[P_{\varepsilon}\right]=$ $\varphi\left(\right.$ ind $\left.p_{\varepsilon}\right)$ (в силу первой части доказательства), а с другой стороны, переход к пределу при $\varepsilon \rightarrow 0$ возможен в силу предложения 9.1. Итак, достаточно доказать справедливость утверждений (a) и (b).

Утверждение (а) следует из гомотопической инвариантности индекса, поскольку символ $p_{\varepsilon}$ изменяется при изменении $\varepsilon$ лишь вне достаточно большого шара $\{|\xi|>R\}$, где $R \simeq 1 / \varepsilon$, и является вне этого шара обратимым.

Утверждение (b) получается следующим образом.

1. Семейства $P_{\varepsilon}$ и $P_{\varepsilon}^{*}$ сильно непрерывны, поскольку они равномерно ограничены и каждое слагаемое, участвующее в их определении в координатных картах на $X$, сильно непрерывно на множестве функций, преобразование Фурье которых имеет компактный носитель.

2. Операторы $f\left(P_{\varepsilon} P_{\varepsilon}^{*}-1\right)$ и $f\left(P_{\varepsilon}^{*} P_{\varepsilon}-1\right)$ для $f \in C_{0}\left(X^{\circ}\right)$ компактны и непрерывно зависят от $\varepsilon$. Действительно, компактность очевидна, а непрерывность вытекает из того, что их полные символы в локальных системах координат вместе с производными равномерно непрерывно зависят от $\varepsilon$ на компактных множествах по $\xi$, равномерно ограничены и убывают при $\xi \rightarrow \infty$, а значит, равномерно непрерывно зависят от $\varepsilon$ при всех $\xi$.

Аналогично устанавливается компактность и непрерывность коммутаторов $\left[f, P_{\varepsilon}\right]$ и $\left[f, P_{\varepsilon}^{*}\right]$. Теорема 7.1 доказана.

\section{Список литературы}

1. M.F. Atiyah, "Global theory of elliptic operators", Functional analysis and related topics, Proc. Int. Conf. (Tokyo 1969), Univ. Tokyo Press, Tokyo, 1970, 21-30.

2. Г. Г. Каспаров, "Обобщенный индекс эллиптических операторов", Функи. анализ и его прилож., 7:3 (1973), 82-83; англ. пер.: G. G. Kasparov, "The generalized index of elliptic operators", Funct. Anal. Appl., 7:3 (1973), 238-240.

3. L. Brown, R. Douglas, P. Fillmore, "Extensions of $C^{*}$-algebras and $K$-homology", Ann. of Math. (2), 105:2 (1977), 265-324.

4. I. M. Singer, "Future extensions of index theory and elliptic operators", Prospects in mathematics (Princeton, NJ, 1970), Ann. of Math. Studies, 70, Princeton Univ. Press, Princeton, NJ, 1971, 171-185. 
5. A. Savin, "Elliptic operators on singular manifolds and $K$-homology", $K$-Theory, 34:1 (2005), 71-98.

6. C. Debord, J.-M. Lescure, " $K$-duality for pseudomanifolds with isolated singularities", J. Funct. Anal., 219:1 (2005), 109-133.

7. J.-M. Lescure, Elliptic symbols, elliptic operators and Poincare duality on conical pseudomanifolds, arXiv: math.0A/0609328.

8. V. Nazaikinskii, A. Savin, B.-W. Schulze, B. Sternin, On the homotopy classification of elliptic operators on manifolds with edges, Preprint № 2004/16, Univ. Potsdam, Inst. Math., Potsdam, 2004; arXiv: math.0A/0503694.

9. R. Melrose, F. Rochon, "Index in $K$-theory for families of fibred cusp operators", K-Theory, 37:1-2 (2006), 25-104; arXiv: math.DG/0507590.

10. Б. А. Пламеневский, В.Н. Сеничкин, "О классе псевдодифференциальных операторов в $\mathbb{R}^{m}$ и на стратифицированных многообразиях", Матем. сб., 191:5 (2000), 109-142; англ. пер.: B. A. Plamenevskii, V. N. Senichkin, "On a class of pseudodifferential operators in $\mathbb{R}^{m}$ and on stratified manifolds", Sb. Math., 191:5 (2000), 725-757.

11. V. Nistor, A. Weinstein, P. Xu, "Pseudodifferential operators on differential groupoids", Pacific J. Math., 189:1 (1999), 117-152.

12. R. Lauter, B. Monthubert, V. Nistor, "Pseudodifferential analysis on continuous family groupoids", Doc. Math., 5 (2000), 625-655.

13. R. Melrose, "Pseudodifferential operators, corners, and singular limits", Proceedings of the international congress of mathematicians (Kyoto, Japan, 1990), Math. Soc. Japan, Tokyo; Springer-Verlag, Tokyo, 1991, 217-234.

14. R. Lauter, V. Nistor, "Analysis of geometric operators on open manifolds: a groupoid approach", Quantization of singular symplectic quotients (Oberwolfach, Germany, 1999), Progr. Math., 198, Birkhäuser, Basel, 2001, 181-229.

15. V. Nistor, "Pseudodifferential operators on non-compact manifolds and analysis on polyhedral domains", Spectral geometry of manifolds with boundary and decomposition of manifolds (Roskilde, Denmark, 2003), Contemp. Math., 366, Amer. Math. Soc., Providence, RI, 2005, 307-328.

16. P. Baum, A. Connes, "Geometric $K$-theory for Lie groups and foliations", Enseign. Math. (2), 46:1-2 (2000), 3-42.

17. J.-L. Tu, "La conjecture de Baum-Connes pour les feuilletages moyennables", K-Theory, 17:3 (1999), 215-264.

18. Б. А. Пламеневский, В.Н.Сеничкин, "Представления $C^{*}$-алгебр псевдодифференциальных операторов на кусочно гладких многообразиях", Алгебра и анализ, 13:6 (2001), 124-174; англ. пер.: B. A. Plamenevskii, V. N. Senichkin, "Representations of $C^{*}$-algebras of pseudodifferential operators on piecewise-smooth manifolds", St. Petersburg Math. J., 13:6 (2002), 993-1032.

19. D. Calvo, C.-I. Martin, B.-W. Schulze, "Symbolic structures on corner manifolds", Microlocal analysis and asymptotic analysis (Kyoto, Japan, 2004), Keio Univ., Tokyo, 2005, 22-35.

20. G. Luke, "Pseudodifferential operators on Hilbert bundles", J. Differential Equations, 12:3 (1972), 566-589.

21. В.Е. Назайкинский, А. Ю. Савин, Б. Ю. Стернин, "Псевдодифференциальные операторы на стратифицированных многообразиях. I, II", Дифференц. уравнения, 43:4 (2007), 519-532; 43:5 (2007), 685-696; англ. пер.: V.E. Nazaikinskii, A. Yu. Savin, B. Yu. Sternin, "Pseudodifferential operators on stratified manifolds. I, II", Differ. Equ., 43:4 (2007), 536-549; 43:5 (2007), 704-716.

22. N. Higson, J. Roe, Analytic K-homology, Oxford Math. Monogr., Oxford Univ. Press, Oxford, 2000. 
23. M. Atiyah, V. Patodi, I. Singer, "Spectral asymmetry and Riemannian geometry. III", Math. Proc. Cambridge Philos. Soc., 79:1 (1976), 71-99.

24. A. Connes, N. Higson, "Déformations, morphismes asymptotiques et $K$-théorie bivariante", C. R. Acad. Sci. Paris, Sér. I. Math., 311:2 (1990), 101-106.

25. N. Higson, "On the $K$-theory proof of the index theorem", Index theory and operator algebras (Boulder, CO, USA, 1991), Contemp. Math., 148, Amer. Math. Soc., Providence, RI, 1993, 67-86.

26. В. М. Мануйлов, "Об асимптотических гомоморфизмах в алгебру Калкина", Функи. анализ и его прилож., 35:2 (2001), 81-84; англ. пер.: V. M. Manuilov, "On asymptotic homomorphisms into Calkin algebras", Funct. Anal. Appl., 35:2 (2001), $148-150$.

27. A. Connes, Noncommutative geometry, Academic Press, San Diego, CA, 1994.

28. В. Е. Назайкинский, А. Ю. Савин, Б. Ю. Стернин, Б.-В. Шульце, "Об индексе эллиптических операторов на многообразиях с ребрами”, Матем. сб., 196:9 (2005), 23-58; англ. пер.: V.E. Nazaikinskii, A. Yu. Savin, B. Yu. Sternin, B.-W. Schulze, "On the index of elliptic operators on manifolds with edges", Sb. Math., 196:9 (2005), 1271-1305.

29. V. Nazaikinskii, A. Savin, B.-W. Schulze, B. Sternin, Elliptic Theory on Manifolds with Nonisolated Singularities. III. The Spectral Flow of Families of Conormal Symbols, Preprint № 2002/20, Univ. Potsdam, Institut für Mathematik, Potsdam, 2002.

30. R. Melrose, P. Piazza, "Analytic K-theory on manifolds with corners", Adv. Math., 92:1 (1992), 1-26.

31. P. Baum, R. G. Douglas, "Index theory, bordism, and K-homology", Operator algebras and K-theory (San Francisco, 1981), Contemp. Math., 10, Amer. Math. Soc., Providence, RI, 1982, 1-31.

32. J. Roe, "Coarse cohomology and index theory on complete Riemannian manifolds", Mem. Amer. Math. Soc., 104:497 (1993), 1-90.

33. B. Monthubert, "Groupoids of manifolds with corners and index theory", Groupoids in analysis, geometry, and physics (Boulder, CO, USA, 1999), Contemp. Math., 282, Amer. Math. Soc., Providence, RI, 2001, 147-157.

34. B. Blackadar, " $K$-theory for operator algebras", Mathematical sciences research institute publications, Math. Sci. Res. Inst. Publ., 5, Cambridge Univ. Press, Cambridge, 1998.

35. Л. Хермандер, Анализ линейных дифференциальных операторов с частными производными. Т. III. Псевдодифференциальные операторь, Мир, М., 1987; пер. с англ.: L. Hörmander, The analysis of linear partial differential operators. III. Pseudodifferential operators, Grundlehren Math. Wiss., 274, Springer, Berlin, 1985.

B. Е. НАЗАЙкинский (V. Е. NAZAikinskiı)

Поступило в редакцию Московский государственный университет 06.02.2006 им. М.В.Ломоносова

А. Ю. САвин (А. Yu. Savin)

Независимый московский университет

E-mail: antonsavin@mail.ru

Б. Ю. Стернин (B. Yu. Sternin)

Московский институт электронного машиностроения

E-mail: sternin@mail.ru 